\title{
Modernes Dysphagiemanagement in der neurologisch-neurochirurgischen Frührehabilitation
}

Neurol Rehabil 2016; 22(3): 231-250

(C) Hippocampus Verlag 2016

DOI 10.14624/NR150816.006

\author{
C. Ledl, M. Mertl-Rötzer, M. Schaupp
}

\begin{abstract}
Zusammenfassung
Die Inzidenz von Schluckstörungen in der neurologisch-neurochirurgischen Frührehabilitation (NNFR) variiert in Abhängigkeit der Grunderkrankung von 51\%-91\%. Häufige Komplikationen sind Aspirationspneumonien und Mangelernährung, die eine erhöhte Mortalität und ein schlechteres funktionelles Outcome nach sich ziehen. Die Diagnostik besteht aus orientierenden Screeningverfahren, klinischer Fachuntersuchung und instrumentellen Untersuchungen wie FEES (flexible endoskopische Evaluation des Schluckens), Videofluoroskopie und hochauflösender Ösophagusmanometrie. Therapieansätze gliedern sich nach kausalen, kompensatorischen und adaptativen Verfahren. Perspektivisch ist zu erwarten, dass neuromodulative Verfahren als Ergänzung der traditionellen Therapie Einsatz finden werden, um den Behandlungserfolg zu steigern. Interdisziplinär spielen Maßnahmen wie Atmungstherapie, individualisiertes Ernährungsmanagement und medikamentöses Sekretmanagement eine wichtige Rolle.
\end{abstract}

Schlüsselwörter: neurogene Dysphagie, neurologisch-neurochirurgische Frührehabilitation, Diagnostik, Therapie

\section{Inzidenz der Dysphagie und deren strukturelle Bedeutung}

Das Auftreten einer Dysphagie ist stark abhängig von der zugrundeliegenden Erkrankung und variiert zwischen 51-91\%. Für die Gruppe der Schlaganfallpatienten wird die Inzidenz der Dysphagie mit 29-67\% angegeben. Werden apparative Verfahren zur Diagnostik verwendet, beträgt die Inzidenz bis zu 80 \% [66]. Das Vorliegen einer Schluckstörung bei Schlaganfallpatienten erhöht die Mortalität um das Dreifache. Komplikationen wie Mangelernährung, Dehydration und Infektionen der unteren Atemwege sind häufig [93]. Schlaganfallpatienten haben ein 3-fach höheres, bei nachgewiesener Aspiration sogar 11,5-fach erhöhtes Pneumonierisiko [66]. Die Inzidenz der Dysphagie bei Schädel-Hirnverletzungen wird mit $25-78 \%$ angegeben [85], bei schwerem Schädel-HirnTrauma bis zu 93\% [38]. Potentiell lebensbedrohliche Komplikation wie Mangelernährung, Dehydration, Aspirationspneumonien sind auch hier häufig. 27\% einer retrospektiven Kohortenstudie von 173 SHT-Patienten, die von einer Intensivstation in eine Frührehabilitationseinrichtung (»brain injury unit«) verlegt wurden, waren deswegen schon antibiotisch behandelt worden, weitere $12 \%$ erlitten eine Pneumonie während der Frührehabilitationsbehandlung [38].

Eine multizentrische Erfassung zum Rehabilitationsverlauf von Patienten in der NNFR Phase B in 16 neurologischen Frührehabilitationseinrichtungen in
10 Bundesländern [76] schloss innerhalb eines Monats 754 Patienten ein, die in diesem Monat aus der NNFR entlassen wurden. Das Alter der Patienten betrug im Mittel 68 Jahre, 25,5\% der Patienten waren bei Aufnahme in die Frührehabilitation beatmet, 59,1\% waren enteral oder parenteral ernährt. Bei $61,7 \%$ bestand eine beaufsichtigungspflichtige Schluckstörung, 41,5\% waren mit einem absaugpflichtigen Tracheostoma versorgt. Damit leiden fast zwei Drittel der Patienten der NNFR an einer höhergradigen Dysphagie.

\section{Critical Illness Dysphagie und Post-Extubation Dysphagia}

In einer retrospektiven Kohortenstudie (2008-2010; $\mathrm{N}=2.484$ ) an Erwachsenen wurde das Auftreten einer Dysphagie nach längerer Intubation (post-extubation dysphagia) untersucht [64]. Die Diagnose einer Dysphagie wurde klinisch durch eine »bedside swallow evaluation « gestellt, wobei nur 25\% aller beatmeten Patienten hinsichtlich der Schluckstörung untersucht wurden. Bei den nicht neurologisch Erkrankten fand sich in 84\% ( $\mathrm{N}=374)$ eine Dysphagie unterschiedlicher Ausprägung: mild, moderat bzw. schwer betroffen waren $44 \%$ $(\mathrm{N}=195), 23 \%(\mathrm{~N}=103)$ bzw. $17 \%(\mathrm{~N}=76)$. Die Zeitdauer der Intubation (>7 Tage) und die Notwendigkeit einer Reintubation waren statistisch signifikante Risikofaktoren, eine schwere Dysphagie zu bekommen (adjustierte odds ratio $[A O R]=2,84)$. Darüber hinaus war eine schwere Dysphagie post extubationem signifikant assoziiert 
Modern management of dysphagia in early neurological and neurosurgical rehabilitation

C. Ledl, M. Mertl-Rötzer, M. Schaupp

\section{Abstract}

The incidence of swallowing disorders in early neurological and neurosurgical rehabilitation varies from $51 \%$ to $91 \%$ depending on the underlying disease. Aspiration pneumonia and malnutrition are frequent complications leading to increased mortality and reduced functional outcome. Diagnostic procedures comprise screenings, clinical dysphagia examinations and instrumental techniques as FEES (flexible endoscopic evaluation of swallowing), VFSS (videofluoroscopic swallow study) and high resolution esophageal manometry. Therapeutic approaches include restorative, compensatory and adaptive techniques. It is to be expected that neuromodulative approaches will be a complement to traditional therapy in the future to improve treatment outcome. Important interdisciplinary approaches in dysphagia management are respiratory therapy, individualized nutritional management and pharmacological secretion management.

Keywords: neurogenic dysphagia, early rehabilitation, diagnostics, therapy

Neurol Rehabil 2016; 22(3): 231-250

(C) Hippocampus Verlag 2016 einer kompletten Normalisierung des Schluckakts, die laryngeale Sensibilitätsstörung erholte sich bei $75 \%$ der Betroffenen.

Angesichts der nachweislich hohen Inzidenz von Dysphagie bei Intensivpatienten sollte die Aufmerksamkeit und Wahrnehmung für Dysphagie bei intensivmedizinischem Fachpersonal in Deutschland erfasst werden. Auf dem 24. Symposium für Intensivmedizin \& Intensivpflege 2014 in Bremen wurde mittels einer PaperpencilBefragung eine Punktprävalenzstudie durchgeführt. Von den 4.664 Teilnehmern (46\% Pflege, 54\% Ärzte und Therapeuten) betrug der Rücklauf nur $8 \%$. Dies bestätigt die Vermutung, dass das Bewusstsein für das Vorliegen dysphagischer Störungen auf deutschen Intensivstationen gering ist. Darüber hinaus bestand ein ausgeprägter Berufsgruppenunterschied in der Aufmerksamkeit für Dysphagie: $80 \%$ der Antwortbögen kamen von den Pflegekräften. Die Häufigkeit des Vorhandenseins einer Dysphagie wurde mit 30-50\% als relativ hoch eingeschätzt. Dennoch durften nur 5 bzw. 3,9\% der Patienten nicht essen oder trinken (Datenpräsentation auf dem DGD-Kongress in Hamburg 2015). Insofern kommt dem Dysphagiemanagement in der NNFR eine wesentliche Bedeutung zu.

Häufig wird die primäre Schluckstörung durch weitere neurologische oder neuropsychologische Symptome, v.a. durch schwere Bewusstseins- oder Aufmerksamkeitsstörungen, und durch unterschiedlichste Begleiterkrankungen aggraviert. Dies macht eine Behandlung im interdisziplinären Team erforderlich. Diesem gehören neben den in Diagnostik und Therapie spezialisierten Schlucktherapeuten auch im Umgang mit Dysphagien erfahrene Pflegetherapeuten, Atmungstherapeuten, Physio- und Ergotherapeuten sowie Neuropsychologen an. Im Hinblick auf die Diagnostik und die Behandlung der Grund- und Begleiterkrankungen ist eine hohe Expertise im neurologisch-ärztlichen Bereich erforderlich, die oftmals die Kooperation zusätzlicher Disziplinen erfordern wird (Anästhesie, Chirurgie, Gastroenterologie, HNO/Phoniatrie, Pulmologie, Radiologie). Strukturelle Voraussetzungen sind das Vorhalten entsprechender personeller Ressourcen mit Erfahrung in Schluckdiagnostik und -therapie sowie spezifisch räumlich-apparative Ausstattung (instrumentelle Diagnostik, supervidierte Essensbereiche, Geräte zur Durchführung maschineller Atmungstherapie). Ebenso müssen relevante Prozesse (diagnostische und therapeutische Standards, diätetische Anpassungen, Sonden- und Trachealkanülenmanagement) ausgestaltet werden. Letztlich soll ein modernes Dysphagiemanagement in den Einrichtungen der NNFR auch eine Messung der Ergebnisqualität umfassen (z. B. Raten für Dekanülierung, oralen Kostaufbau, Entwöhnung Ernährungssonden), um eine kontinuierliche Qualitätskontrolle und Verbesserung der Behandlung sicherzustellen. 


\section{Behandlungspfad Klinische Dysphagiediagnostik}

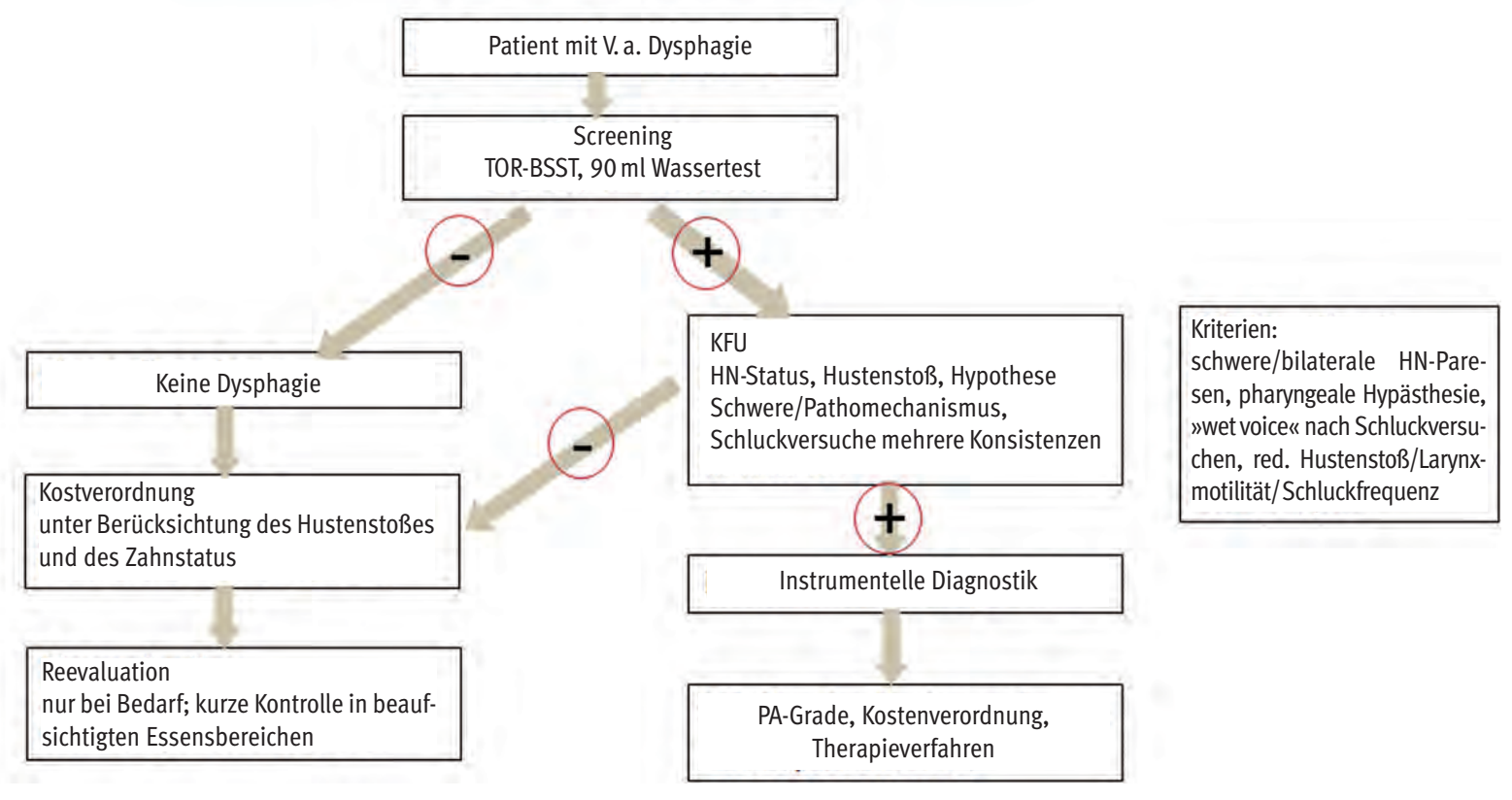

Abb. 1: Behandlungspfad Klinische Fachdiagnostik (Schön Klinik Bad Aibling)

\section{Klinische und instrumentelle Diagnostik der Dysphagien}

Ziel der Dysphagiediagnostik ist die Analyse der Patientengefährdung durch Aspiration, die Festlegung des Trachealkanülenmanagements, der oralen Ernährbarkeit sowie der Fähigkeit, Medikamente zu schlucken. Die Diagnostik läuft, wie in Abbildung 1 dargestellt, gestuft ab. Sie beginnt mit einem Screening, das entweder eine Dysphagie ausschließt oder über die Indikation einer klinischen Fachuntersuchung (KFU) entscheidet. In der KFU werden anhand klinischer Prädiktoren die orale Ernährbarkeit der Patienten wie auch der instrumentelle Untersuchungsbedarf festgelegt.

\section{Screeningverfahren}

Screeningverfahren sind einfach durchzuführende Kurztests, die Aufschluss über die Aspirationsgefährdung geben und über die Zuweisung zur Fachdiagnostik entscheiden. Sie werden von geschulten Pflegetherapeuten oder Sprach-/Schlucktherapeuten durchgeführt. Zur Anwendung kommen Wassertests, die durch zusätzliche Beurteilungskriterien zu Stimmqualität, Hustenstoß, Würgereflex und Vorhandensein einer Sprech- und Stimmstörung ergänzt werden.

Die Testvalidität der Verfahren ergibt sich aus ihrer jeweiligen Sensitivität und Spezifität und wird durch den Vergleich mit objektiven Testverfahren (hier: Schluckendoskopie und/oder Videofluoroskopie) ermittelt. Die- ses methodische Vorgehen ist Auswahlkriterium für die im Folgenden genannten Verfahren. Die Sensitivität gibt an, wie hoch der Anteil der richtig positiv klassifizierten Patienten ist, die Spezifität zeigt den Anteil der richtig negativ klassifizierten Patienten an. Beide Werte sollen über $70 \%$ liegen.

Alle Aspirations-Screenings wurden an akuten Schlaganfallpatienten entwickelt, sodass zu überprüfen ist, ob die Verfahren auf Patienten in der NNFR übertragbar sind. Eine Reliabilitätsanalyse bei postakuten Patienten in der NNFR ergab sehr gute Sensibilitätswerte der Prozeduren bei allerdings deutlich unterdurchschnittlicher Spezifität, sodass von einer überdurchschnittlich hohen Rate falsch positiver Screeningergebnisse ausgegangen werden muss [56]. In der klinischen Praxis bedeutet dies, dass auffällige Testergebnisse wenig Aussagekraft besitzen, bei Patienten mit unauffälligem Screening aber eine Dysphagie mit großer Wahrscheinlichkeit ausgeschlossen werden kann.

In der Praxis finden folgende Tests nach oben genannten Kriterien Anwendung:

- Daniels-Test [20]

- 90-ml-Wassertest [88]/ Yale-Swallow Protocol [89]

- TOR-BSST $^{\circledR}$ (Toronto Bedside Swallowing Screening Test) [67]

- GUSS (Gugging Swallowing Screen) [95]

- Schluckprovokationstests

Beim Daniels-Test werden Wasserschlucke von 5, 10 und $20 \mathrm{ml}$ je $2 \mathrm{x}$ durchgeführt. Der Test gilt als positiv, wenn 
2 von 6 Kriterien erfüllt sind: Dysarthrie, Dysphonie, reduzierter willkürlicher Hustenstoß, reduzierter Würgreflex, Husten nach dem Schluck, Stimmveränderung nach dem Schluck. Der Test wurde an 59 Personen validiert (Sensitivität $=92 \%$, Spezifität $=67 \%$ bei akuten Schlaganfallpatienten).

Beim 90-ml-Wassertest/Yale Swallow Protocol sollen Patienten $90 \mathrm{ml}$ Wasser ohne Unterbrechung aus einem Glas trinken. Der Test ist positiv, wenn das Volumen nicht bewältigt wird, die Stimmqualität sich verändert oder der Patient hustet oder sich räuspert. Das Verfahren ist an 3.000 Personen (darunter 936 neurologische Patienten) validiert, seine Sensitivität liegt bei 96,5\%. Ein negatives Testergebnis kann deshalb zum klinischen Ausschluss einer Schluckstörung verwendet werden. Allerdings ist die Spezifität gering, die Rate Falsch-Positiver beträgt 51,3\%. Angesichts des großen Wasservolumens sollte der Test nicht ohne Vortest zur Risikoabschätzung durchgeführt werden. Die im »Yale Swallow Protocol " genannten Ausschlusskriterien sind: nicht ausreichende Wachheit/Aufmerksamkeit, vorbestehende Sondenernährung, Deoralisierung sowie Einschränkungen dünnflüssiger Konsistenzen, Oberkörperhochlagerung <30 Grad, Trachealkanüle. Zudem wird vor Testdurchführung eine orientierende kognitive und fazio-orale Untersuchung (Orientierung zu Person, Ort und Zeit, Lippenschluss, linguale Motilität und faziale Symmetrie) angeraten.

Der TOR-BSST ${ }^{\circledast}$ (Sensitivität $91 \%$, Spezifität $67 \%$ ) wurde an 59 Schlaganfallpatienten validiert. Patienten sollen 10x $5 \mathrm{ml}$ Wasser schlucken und aus einer Tasse trinken. Beurteilt werden linguale Paresen, Stimmqualität, Drooling (Wasser-/Speichelauslauf aus dem Mund) und Husten/Räuspern beim Schlucken. Der Test ist positiv, sobald ein Item positiv ist. Die Testvalidität entspricht der des Daniels Tests. Vorteile des TOR-BSST sind eine strikte Standardisierung und Schulung.

Das GUSS (Sensitivität 100\%; Spezifität 50\% bzw. 69\%; validiert an zwei Gruppen von 19 und 30 Patienten) besteht aus zwei Teilen, wobei in der Vortestung Vigilanz und Reinigungsfunktion geprüft werden:

A Speichelschlucke sowie Beurteilung von Vigilanz, willkürlichem Hustenstoß und Räuspern,

B Schluckversuche mit angedicktem Wasser, Wasserschlucke mit verschiedenen Volumina $(3 \mathrm{ml}, 5 \mathrm{ml}$, $10 \mathrm{ml}, 50 \mathrm{ml}$ ) und Brot.

Beurteilungskriterien bei den Schluckversuchen sind Schluckfähigkeit, Stimmveränderung, Drooling und Husten. Dieses Verfahren testet als einziges validiertes Screening mehrere Konsistenzen unter der Annahme zunehmender Schwierigkeit des Schluckakts. Es ist unbewiesen, ob das Schlucken fester Konsistenzen tatsächlich schwieriger ist als das von Flüssigkeiten und damit die aus dem Testverlauf resultierenden Ernährungsempfehlungen adäquat sind.
Beim Schluckprovokationstest (Sensitivität $[0,4 \mathrm{ml}]$ 74\%; [2 ml] 49\%; Spezifität $100 \%$ [0,4 $\mathrm{ml}$ und $2 \mathrm{ml}$ ], validiert an 100 Patienten [94, 97]) wird unter Umgehung der oralen Phase ein dünner Katheter (Durchmesser: 4 Charrière) transnasal bis kaudal des Velums vorgeschoben. Über diesen werden 0,4 $\mathrm{ml}$ bzw. $2 \mathrm{ml}$ Wasser in den Oropharynx verabreicht. Der Test gilt als unauffällig, wenn der Patient innerhalb von 3 Sekunden schluckt. Bei akuten Schlaganfallpatienten werden die geforderten Testkriterien (Sensitivität und Spezifität $\geq 70 \%$ ) nur von dem $0,4 \mathrm{ml}$ Schluckprovokationstest erreicht, dessen Sensitivität allerdings im Vergleich zu anderen Verfahren gering ist. Der Vorteil der Schluckprovokation liegt also in der hohen Spezifität des Verfahrens. Die Untersuchung an postakuten Patienten [56] ergab nur für den $2 \mathrm{ml}$ Provokationstest für Flüssigkeiten eine ausreichende Sensitivität $(77,8 \%)$ und Spezifität $(71,4 \%)$, die Spezifität von TOR-BSST und Daniels-Test lagen zum Teil erheblich unter den bei Akutpatienten gemessenen Ergebnissen.

Die Auswahl des geeigneten Screeningverfahrens muss in der NNFR aufgrund der hohen Vigilanz- und Leistungsunterschiede individualisiert erfolgen. Für vigilante und dysphagisch weitgehend unauffällige Patienten wird sich der $90 \mathrm{ml}$ Wassertest zum Ausschluss einer Dysphagie eignen. Sollte die häufig erforderliche Stimmprobe z.B. aufgrund einer Tracheotomie nicht möglich sein, kann auf den 2-ml-Provokationstest zurückgegriffen werden. Der TOR-BSST besticht durch die einfache und schnelle Durchführbarkeit.

\section{Klinische Fachdiagnostik}

In verschiedenen klinischen Routinen wird nach positivem Screeningergebnis die Durchführung einer instrumentellen Untersuchung gefordert. Angesichts der hohen Rate falsch positiver Screeningergebnisse sollte eine klinische Fachdiagnostik vorgeschalten werden. Diese umfasst die Überprüfung des Hirnnervenstatus, des willkürlichen und reflektorischen Hustenstoßes sowie Schluckversuche unter Erprobung mehrerer Konsistenzen und Kompensationsstrategien.

\section{Kaudaler Hirnnervenstatus}

Die Überprüfung des kaudalen Hirnnervenstatus beinhaltet die Ruhebeobachtung, die Palpation fazial, oral und kranial sowie die Überprüfung der willkürlichen und reflektorischen Funktionen und der Sensibilität intraoral und pharyngeal. Es wird eine Hypothese zur Schluckfähigkeit abgeleitet und anhand der nachfolgenden Schluckversuche verifiziert [57]. Die Überprüfung des kaudalen Hirnnervenstatus erlaubt eine Differenzierung nach Läsionen des 1. und 2. Motoneurons und ist therapieleitend. Im Allgemeinen wird nach zentralen Paresen die Entwicklung einer Spastik erwartet, sodass 


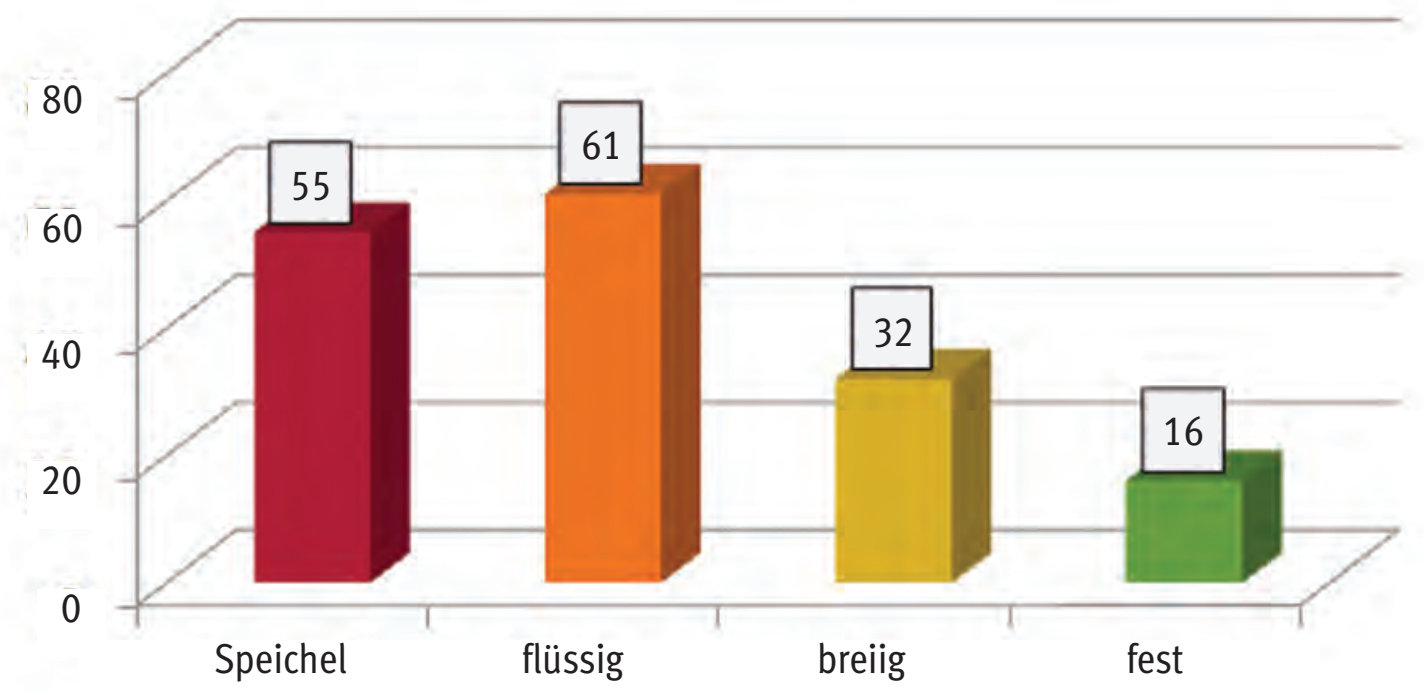

Abb. 2: FEES: Aspiration (\%) in Abhängigkeit von den getesteten Konsistenzen in 100 sukzessiven FEES-Untersuchungen (2008). Aspiration $=$ Wert auf PA-Skala (Rosenbeck) $\geq 6$

Kräftigungs- und Belastungsübungen kontraindiziert sein können. Bei peripheren Paresen hingegen steht die Verhinderung der Muskelatrophie im Vordergrund, die am ehesten durch Vibrationsbehandlungen, Kräftigungs- und Widerstandübungen erreicht werden kann.

Zungen- und Larynxmotilität sowie deren Koordination beim Schlucken werden mit Hilfe des Schluckkontrollgriffs beurteilt. Der Zeigefinger wird auf den Mundboden, der Mittelfinger auf das Hyoid (Zungenbein), der Ringfinger auf den Schildknorpel und der kleine Finger auf den Ringknorpel gelegt [6]. Damit lassen sich schwere Motilitätsstörungen des Larynx erfassen, differenziertere Aussagen etwa zur Ventralisation des Hyoids sind der videofluoroskopischen Analyse vorbehalten.

Ein eingeschränkter Hustenstoß ist ein signifikanter Prädiktor für Dysphagien und pulmonale Komplikationen. Ein reduzierter reflektorischer Hustenstoß resultiert nach peripheren Stimmlippenparesen infolge des abgeschwächten subglottischen Druckaufbaus und/ oder nach Paresen der exspiratorischen Muskulatur und wird häufig nach Läsionen der Medulla oblongata, bei Patienten mit neurodegenerativen Erkrankungen sowie bei Patienten mit Polyneuropathien oder -myopathien beobachtet. Die orale Ernährung mit festen Konsistenzen wird bei Patienten mit reduziertem reflektorischem Hustenstoß aufgrund der Erstickungsgefahr nicht empfohlen.

\section{Penetration, Aspiration}

Das Eindringen von Bolus oder Speichel in den Kehlkopfeingang (aditus laryngis) wird als Penetration bezeichnet. Unter Aspiration wird das Vordringen dieser Materialien in den subglottischen Bereich der Luftröhre verstanden. Diese Ereignisse werden nach Schweregraden differenziert, wobei die silente Aspiration - also eine Aspiration ohne Auslösung eines Hustenstoßes - die schwerste, aber auch häufigste Form darstellt. Weitreichende Verbreitung hat die Penetrations-Aspirations-Skala nach Rosenbek et al. [81] gefunden. Unterschieden werden ferner prä-, intra- und postdeglutitive Aspirationstypen.

\section{Austestung von Konsistenzen}

Die Schwere von Aspiration und Penetration variiert in Abhängigkeit der angebotenen Konsistenzen. Es ist daher sinnvoll, mehrere Konsistenzen - ggf. auch unter Anwendung verschiedener Kompensationstechniken $\mathrm{zu}$ erproben. In einer Auswertung von 100 sukzessiven endoskopischen Schluckuntersuchungen aspirierten Patienten mit neurogenen Dysphagien Wasser und Speichel am häufigsten, breiige und feste Konsistenzen deutlich seltener (siehe Abbildung 2). Die klinische Austestung wird meist mit breiigen Konsistenzen begonnen und mit flüssigen, dickflüssigen und - suffizienter Hustenstoß vorausgesetzt - festen Konsistenzen fortgeführt.

Ein Goldstandard der klinischen Untersuchung existiert nicht, ihre Validität wird uneinheitlich beurteilt. Daniels et al. [21] fanden in einer der jüngsten Untersuchungen eine hohe Interrater-Reliabilität sprachtherapeutischer klinischer Bewertungen, betonen aber die Notwendigkeit der Standardisierung von ScreeningItems. Gute Interrater-Reliabilität wurde bei Items zur Beurteilung von Schluckversuchen erzielt. Ausreichende Reliabilität wurde bei Bewertungen von Items zu begleitenden Sprechstörungen oder zur Stimmqualität (»wet voice«) erreicht. 
Klinische Aspirationszeichen sind:

- Husten, Räuspern. Ein fehlender Hustenstoß ist jedoch keine Garantie für einen sicheren Schluckakt, ca. 25\% aller akuten Schlaganfallpatienten aspirieren still.

- Eingeschränkter Zungendruck nach palatal [87] sowie orale Residuen von Speichel oder Bolus (Zunge, Gaumen, Wangentaschen). Residuen weisen auf eine intraorale Hypästhesie, muskuläre Schwäche oder/ und Neglekt-Symptomatik hin.

- Reduzierte Larynxmotilität (Elevation und/ oder Ventralisation) weist auf eine unzureichende Öffnung des OÖS (oberen Ösophagus-Sphinkters) hin; in der Folge kann Bolus nicht oder nur anteilig in den Ösophagus eingeschluckt werden, der pharyngeal verbleibende Bolus(-anteil) wird mit hoher Wahrscheinlichkeit postdeglutitiv aspiriert [74].

- Reduzierte Schluckfrequenz ist assoziiert mit oropharyngo-laryngealen Speichelresiduen. Angaben zur normalen Schluckfrequenz variieren zwischen verschiedenen Untersuchungen und nach Alter. Afkari [2] gibt die normale Schluckfrequenz mit 1,32 Schlucken/Minute an; Tanaka et al. [92] betonen Unterschiede zwischen jüngeren und älteren Probanden (o,68 Schlucke/Min. vs. o,16 Schlucke/Min.).

- Nachschlucken: Nachschlucke werden zur pharyngolaryngealen Reinigung benötigt und können Hinweise geben auf reduzierte Konstriktionskraft und/ oder Larynxmotilität, Zenker-Divertikel, ösophageale Transportstörungen oder gastroösophagealen Reflux.

- Stimmqualität: feuchte postdeglutitive Stimmqualität ist Ausdruck laryngealer Retentionen. Klare Stimmqualität nach dem Schlucken ist ein guter Indikator, dass keine Schluckstörung vorliegt [96]. Fernerhin sind Dysphonie und Aphonie häufig durch ein- oder beidseitige Stimmlippenparesen bedingt, die zu einer Dysfunktion des glottalen Schließreflexes führen und damit die intradeglutitive Aspiration begünstigen können.

- Erschwerte oder nicht vorhandene Auslösbarkeit des Würgreflexes findet sich auch in der Normalpopulation $[55,22]$ und eignet sich nicht zur Differentialdiagnostik; periphere Paresen des Gaumensegels deuten auf eine Hirnstammläsion hin.

- Reduzierte pharyngeale Sensibilität (Kontakt mit Spatel an Pharynxhinterwand) ist ein besserer Aspirationsprädiktor als ein fehlender Würgreflex [22].

- Feuchtes Tracheostoma: meist Zeichen von Speichelaspiration; Aspirationsnachweis bei Austritt von Bolus aus dem Tracheostoma.

- Alter >80 Jahre oder Atemfrequenz >25/Minute oder Baseline der Sauerstoffsättigung $\leq 94 \%$ bei Patienten mit COPD $[19,86]$.
Die in der NNFR relevantesten klinischen Kriterien sind in Abbildung 1 (Behandlungspfad) gelistet.

Darüber hinaus nennen Langmore et al. [54] weitere Faktoren, die signifikant zur Entwicklung von Aspirationspneumonien beitragen: schlechte Mundhygiene sowie nicht-selbstständige Mundpflege, Sondenernährung, nicht-selbstständige Nahrungsaufnahme, Multimorbidität und eingeschränkter Aktivitätslevel. Brogan et al. [10] betonen den starken Zusammenhang zwischen respiratorischen Infektionen und Immobilität. Die Ergebnisse dieser Studie betonen die Notwendigkeit des interdisziplinären Managements dysphagischer Patienten mit ausreichender Durchführung pflege-, physiound atemtherapeutischer Maßnahmen.

\section{Aspirationsbeurteilung bei tracheotomierten Patienten: Methylenblau-Test und modifizierter Methylenblau-Test}

Bei tracheotomierten Patienten werden in Ergänzung zur klinischen Diagnostik der Methylenblau-Test und der modifizierte Methylenblau-Test (mMBT) durchgeführt. Es werden bei entblockter Trachealkanüle (TK) vier Tropfen blaue Lebensmittelfarbe (1\% Lösung) auf die Zunge gegeben, der Patient zum Speichelschluck aufgefordert und im Anschluss tracheal abgesaugt. Cameron et al. [15] fanden durch positive Absaugtests eine Speichelaspiration bei $69 \%$ ihrer Patienten (Beobachtungszeitraum $48 \mathrm{~h}$, Absaugtests alle vier Stunden). Beim modifizierten Methylen-Blau Test sollen die Patienten bei entblockter TK angefärbten Bolus schlucken. Aspiration wird durch nachfolgendes Absaugen überprüft. Vergleiche instrumenteller Untersuchungsergebnisse mit denen des mMBT $[8,26,71]$ zeigen, dass die Sensitivität des mMBT im Zufallsbereich liegt. Klinisch bestätigt also ein positiver mMBT die Aspiration und wird deshalb zur Bestätigung des Aspirationsverdachts klinisch eingesetzt, auch wenn ein negativer mMBT nicht aussagekräftig ist.

\section{Instrumentelle Diagnostik}

Angesichts der Häufigkeit der silenten - und damit der klinischen Untersuchung schwer zugänglichen - Aspiration müssen in der Dysphagiediagnostik instrumentelle Untersuchungstechniken zum Einsatz kommen. Zudem kann die Pathophysiologie der pharyngealen und ösophagealen Phase nur instrumentell geklärt werden.

Der Goldstandard umfasst die Videofluoroskopie/ Röntgenkinematographie und die flexible endoskopische Untersuchung sowie die Tracheo- und Bronchoskopie. Die hochauflösende pharyngo-ösophageale Manometrie wird ergänzend durchgeführt.

\section{Videofluoroskopie (VFSS: videofluoroscopic swallowing study)}

Dynamische Durchleuchtung des Mund-, Rachenraums und des oberen Ösophagus [60, 29, 101]. Die Bildfrequenz der Videofluoroskopie beträgt im europäischen Video-Standard 25 Bilder/Sekunde, d.h. alle 40 Mil- 
lisekunden wird ein Bild aufgezeichnet. Es wird Kontrastmittel verschiedener Konsistenz zum Abschlucken verabreicht und die orale, pharyngeale und ösophageale Passage beurteilt. Der Strahlengang wird zuerst seitlich, zur Bestimmung von Asymmetrien gegebenenfalls auch anterior-posterior geführt (Abb. 3, 4). Die Untersuchung wird in Kooperation von einem Radiologen und einem Sprach-/Schlucktherapeuten durchgeführt. Aufgrund der Strahlenbelastung muss die Indikation zur VFSS eng gestellt werden und die Durchführungsmodalitäten dem ALARA Prinzip (as low as reasonably achievable) entsprechen. Laut einer Studie an 230 Erwachsenen [102] beträgt die effektive Strahlenbelastung während der VFSS im Median 0,2 mSv bei einer durchschnittlichen Durchleuchtungsdauer von 171 Sekunden.

\section{Kontraindikationen:}

- absolut: Allergie gegen Kontrastmittel

- relativ: medizinisch instabile, schläfrige oder agitierte Patienten, die nicht auf Kommando schlucken (Strahlenbelastung), unsichere Positionierung in der Durchleuchtungseinheit.

In Abhängigkeit von der klinischen Fragestellung werden verschiedene Konsistenzen (flüssig, dickflüssig, breiig und fest) ausgetestet. Konsistenzmodifikationen werden erreicht durch Andicken des flüssigen Kontrastmittels oder Beimischung des Kontrastmittels, auch werden Bariumsulfat-Gelatinekugeln verwendet. Normwerte wurden von Kendall et al. [47], Johnson et al. [44] und Hannig [37] vorgelegt und ermöglichen vergleichende Messungen. Qualitative Auswertungen erfolgen mittels individueller Protokollbögen, ein validierter Standard existiert nicht. Hind et al. [40] konnten nachweisen, dass Beurteiler-Trainings zu einer signifikanten Verbesserung der Reliabilität von Penetrations-/Aspirationsbewertungen führen.

\section{Flexible endoskopische Schluckuntersuchung (FEES: flexible} endoscopic evaluation of swallowing)

Die FEES [53, 83] umfasst die Beurteilung der velopharyngo-laryngealen Anatomie und Morphologie, eine Funktions- und Konsistenzenprüfung. Ein flexibles Endoskop wird transnasal über den unteren Nasengang bis zu dessen knöchernem Ende vorgeschoben. Dort Überprüfung der willkürlichen Velumfunktion bei Phonation und der reflektorischen Funktion beim Schlucken. Anschließend wird das Endoskop zirka $1 \mathrm{~cm}$ oberhalb des Epiglottisrands positioniert. In dieser Stellung werden phonatorische und respiratorische Aufgaben zur Beurteilung der Stimmlippenfunktion sowie Schluckversuche mit verschiedenen - zur besseren Erkennung gefärbten - Konsistenzen durchgeführt. Eine Oberflächenanästhesie erfolgt nicht, da eine Sensibilitätsminderung die Kontrolle des Schluckakts beeinträchtigen würde. Zur Passageerleichterung wird

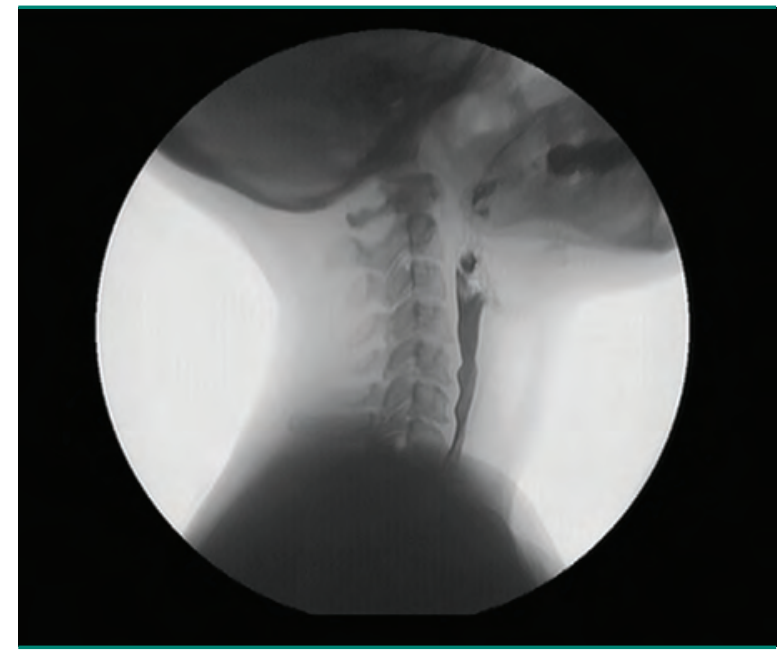

Abb. 3: Videofluoroskopie, lateraler Strahlengang. Unauffälliger Schluckakt mit korrekter Passage des Kontrastmittels in den Ösophagus

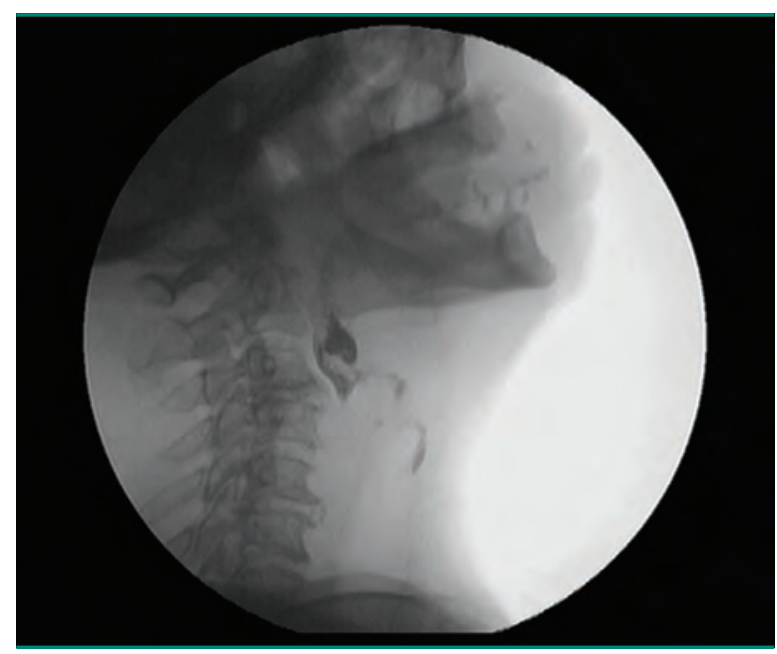

Abb. 4: Videofluoroskopie, lateraler Strahlengang. Oberer Ösophagus-Sphinkter (OÖS) öffnet nicht, das Kontrastmittel läuft nach glottal und wird aspiriert

das Endoskop mit Gleitgel benetzt. Warnecke et al. [98] berichten in einer prospektiven Studie an 300 Patienten keine relevanten untersuchungsassoziierten Komplikationen (vasovagale Reaktion, Laryngospasmus). Hauptvorteile der FEES sind die Beurteilung von Speichel, die Durchführbarkeit auch bei eingeschränkt kooperativen Patienten und die mögliche Prüfung der Sensibilität (siehe Abbildungen 5 und 6). Aviv et al. [3] haben die Überprüfung der pharyngo-laryngealen Oberflächensensibilität durch Luftstöße über den Arbeitskanal des Endoskops vorgeschlagen (FEESST: FEES + sensory testing). Intradeglutitive Aspiration kann aufgrund der Überblendung (»white out«) während der pharyngealen Konstriktion nicht erkannt werden. Ein Ausbildungscurriculum zur Durchführung der FEES wurde von Dziewas et al. [27] entwickelt. 


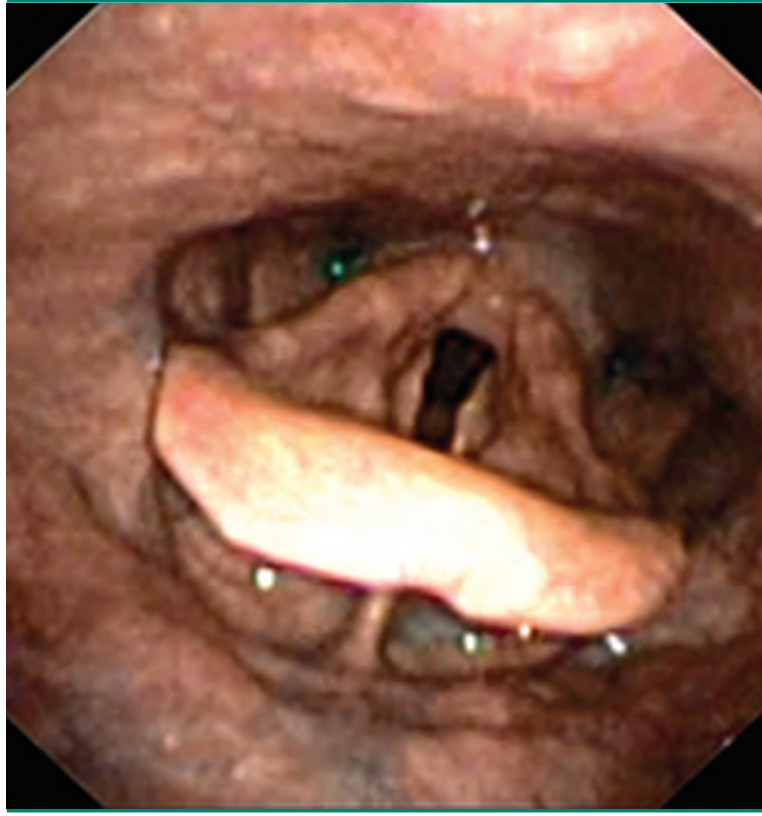

Abb. 5: FEES Normalbefund, Sicht auf Larynx in Respirationsstellung. Die Glottis ist geöffnet, der OÖS ist geschlossen.

\section{Untersuchungsvergleich VFSS - FEES}

Videofluoroskopie und FEES sind komplementäre Untersuchungsmethoden. Penetration und Aspiration lassen sich mit beiden Verfahren differenzieren [18]. Im Vergleich liefert die FEES höhere Penetrationswerte (Penetration-Aspiration-Skala), die VFSS ist in der Beurteilung der Aspirationsmenge überlegen. Einen Überblick der jeweiligen Untersuchungsmerkmale und -anforderungen gibt Tabelle 1.

\section{Hochauflösende Manometrie (HRM) des Pharynx und des} Ösophagus

Ziele der HRM sind der Nachweis der pharyngealen und ösophagealen Druckwelle und Druckstärke (und damit der Boluspropulsion), die zeitliche Analyse von Druckaufbau und -dauer sowie die Bestimmung von Relaxationsbeginn, -amplitude und -dauer des OÖS und UÖS (unterer Ösophagussphinkter).

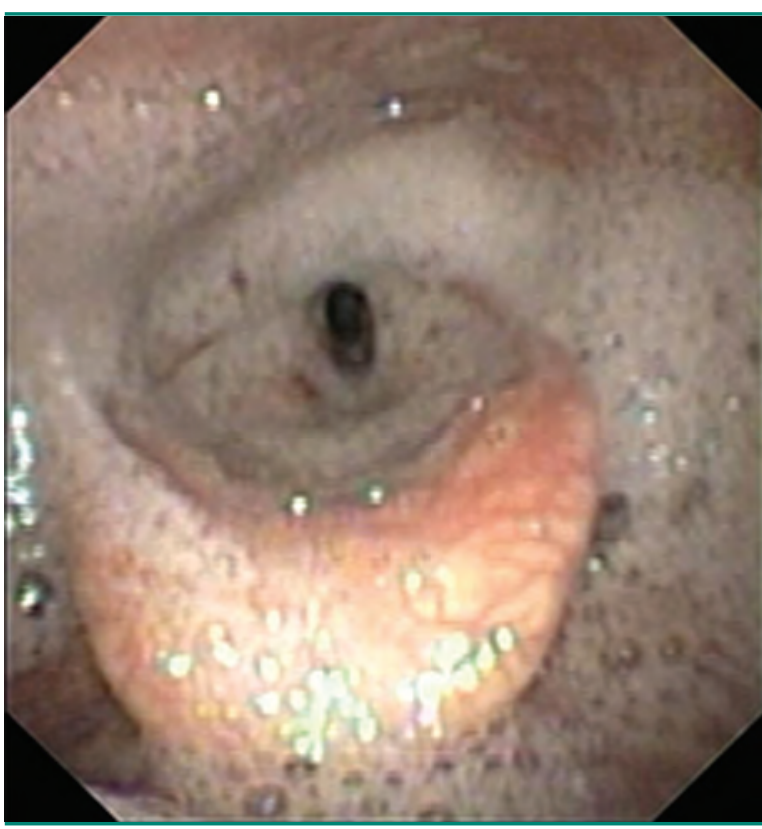

Abb. 6: FEES. Massive Speichelresiduen pharyngo-laryngeal mit ausgeprägter Aspiration.

Die Indikation zur Durchführung wird insbesondere bei V.a. Öffnungsstörung des OÖS, pharyngealer Konstriktionsschwäche, pharyngo-ösophagealer Dyskoordination, nicht strukturell erklärbarer Dysphagie, Achalasie, nicht-kardialem Thoraxschmerz sowie ggf. bei Globusgefühl und Reflux gestellt.

Es wird eine Manometriesonde mit 36 Druckaufnehmern transnasal bis in den Magen vorgeschoben. Ösophageale Normwerte (Chicago Klassifikation) wurden von Kahrilas et al. [45] und Pandolfino et al. [73] erhoben. Pharyngeale Werte liegen für verschiedene Bolusvolumina, Konsistenzen, Altersstufen und Geschlechter vor [13].

Abbildung 7 zeigt auf der y-Achse die farblich kodierten Druckwerte der 36 Sensoren, die von pharyngeal bis gastral reichen, über die Zeit (x-Achse) bei einem Schluckakt mit normaler Ösophagusperistaltik und normaler Öffnung von OÖS und UÖS. Das obere und untere

Tab. 1: FEES und Videofluoroskopie sind komplementäre Verfahren; die klinische Fragestellung bestimmt die Wahl der Untersuchsmethode; +: überlegenes Verfahren

\begin{tabular}{l|l|l}
\hline & FEES & Videofluoroskopie \\
\hline Patienten & alle, auch unkoorperativ, ICU, bedside & Rumpfkontrolle, kooperativ, Schluckakt auf Kommando \\
\hline Konsistenzen & alle & kein Speichel \\
\hline Schluckphasen & pharyngeal, z. T. durch Überblendung eingeschränkt & alle \\
\hline Aspirationstypen & prä- und postdeglutitiv & alle \\
\hline Motorische Steuerung & + Paresenbeurteilung (Velum, Stimmlippen) & $\begin{array}{l}\text { + pharyngeale Konstriktion } \\
\text { + Larynx-, Hyoidmotilität }\end{array}$ \\
\hline Sensibilität & direkte Prüfung & indirekt \\
\hline Beeinträchtigung des Patienten & Gefahr vasovagale Reaktion, Laryngospasmus & Strahlenbelastung \\
\hline
\end{tabular}




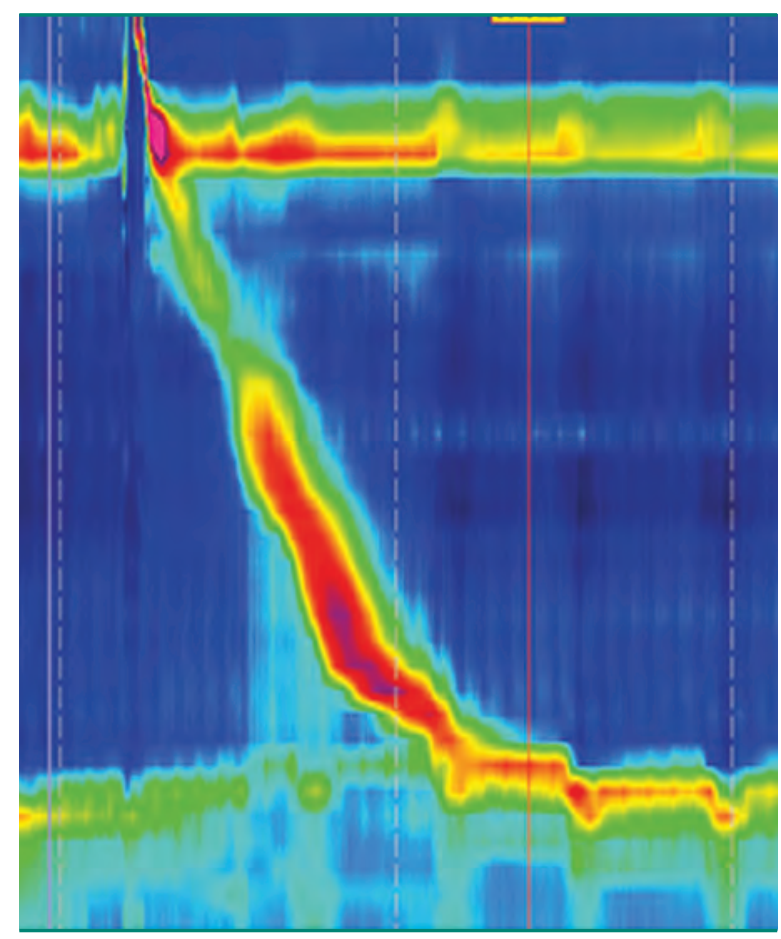

Abb. 7: Hochauflösende Manometrie (HRM), normaler Schluckakt

Farbband zeigt den gemessenen Druck durch OöS und UÖS an. Die Druckamplituden sind von niedrig bis hoch in den Farben blau, grün, gelb und rot kodiert. Die Relaxation des OÖS wird durch die Druckunterbrechung sichtbar, gefolgt von einer sich von pharyngeal nach ösophageal ausbreitenden Druckwelle. Abbildungen 8 und 9 zeigen jeweils Befunde von Patienten mit Wallenberg-Syndrom und klinisch imponierenden schweren Öffnungsstörungen des OÖS; beide Patienten müssen Sekret, Flüssigkeit und Nahrung ausspucken. Während in Abbildung 8 ein deutlich erhöhter Tonus des OÖS vorliegt, imponiert in Abbildung 9 ein hyperkontraktiler distaler Ösophagus bei ausreichender Öffnung des OÖS. Es wird deutlich, dass die HRM einen wichtigen Beitrag zur pathophysiologischen und therapeutischen Differenzierung leistet.

\section{Therapieverfahren}

Ziele des Dysphagiemanagements sind Oralisierung (oraler Kostaufbau bzw. gustatorische Stimulation zur Steigerung der Schluckfrequenz) und Entfernung der Ernährungssonden, Dekanülierung tracheotomierter Patienten und Komplikationsvermeidung. Mittels klinischer und instrumenteller Diagnostikverfahren werden die Realisierbarkeit dieser Ziele und die Möglichkeiten der funktionellen Dysphagietherapie ermittelt. Letztere bestehen aus kausalen, kompensatorischen, adaptativen und neuromodulativen Ansätzen. Der Schweregrad der Dysphagie sowie funktionelle Fortschritte bestimmen das Vorgehen beim Ernährungs- und Tracheal-

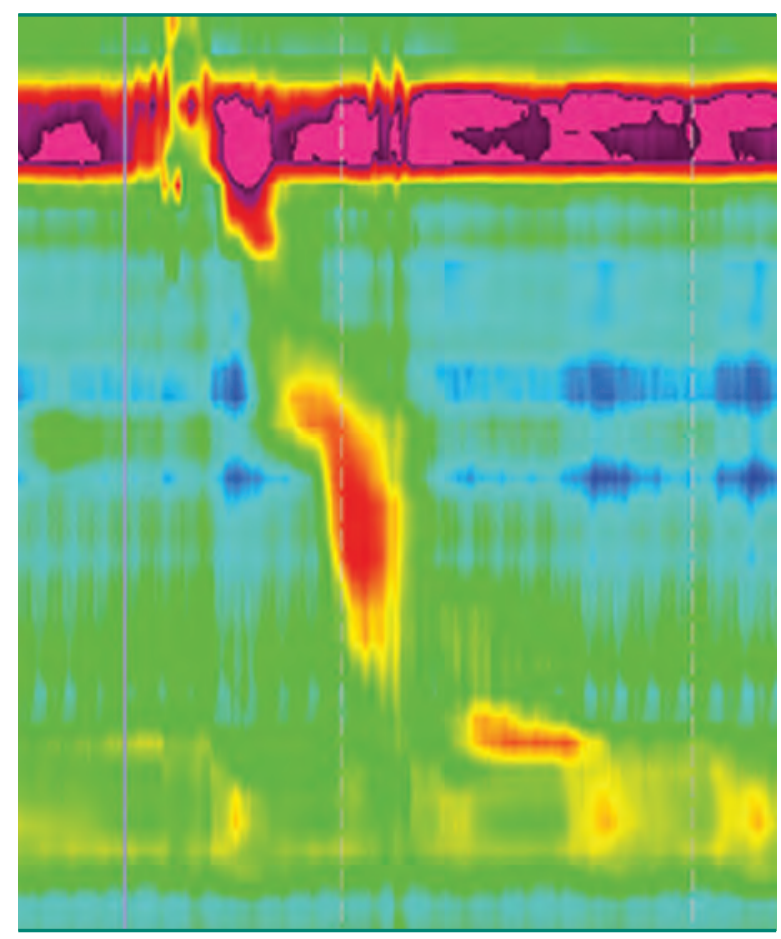

Abb. 8: HRM, Patient mit Wallenberg-Syndrom und fehlender Relaxation und schwerer Tonuserhöhung des OÖS

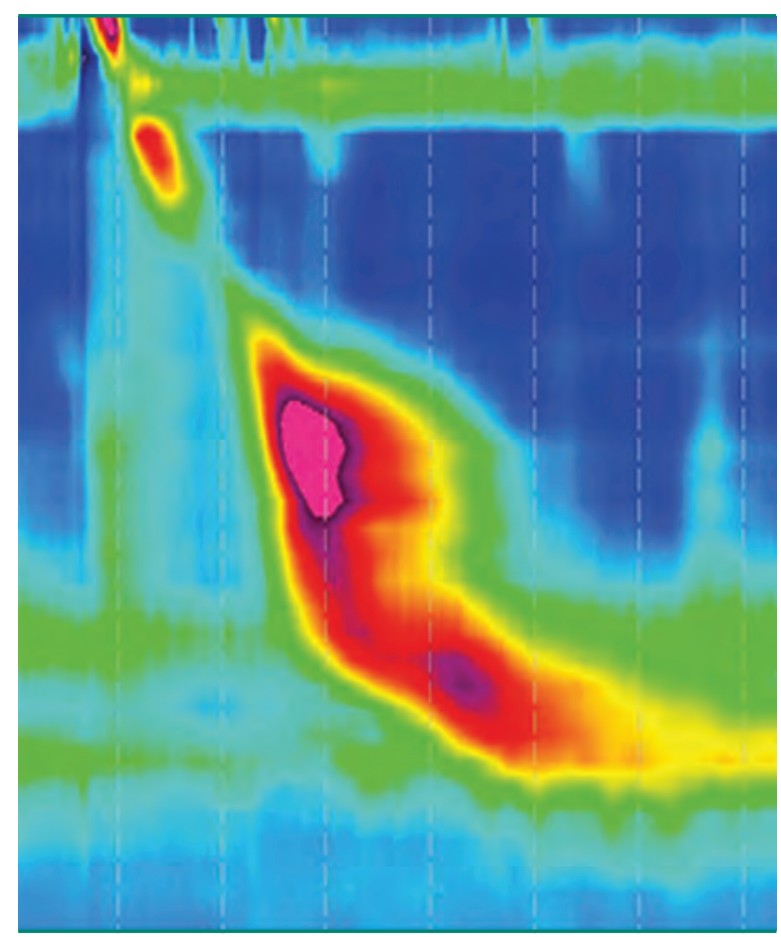

Abb. 9: HRM, Patient mit Wallenberg-Syndrom; ausreichende Relaxation des OÖS, jedoch hyperkontraktiler distaler Ösophagus

kanülenmanagement und werden kontinuierlich ergänzt durch Maßnahmen zur Pneumonieprophylaxe. Dabei sollte keine dieser Maßnahmen für sich allein betrachtet werden, sondern sie stehen in engem Zusammenhang zueinander (siehe Abb. 10). 


\section{Dysphagiemanagement Ziele und Behandlungselemente}

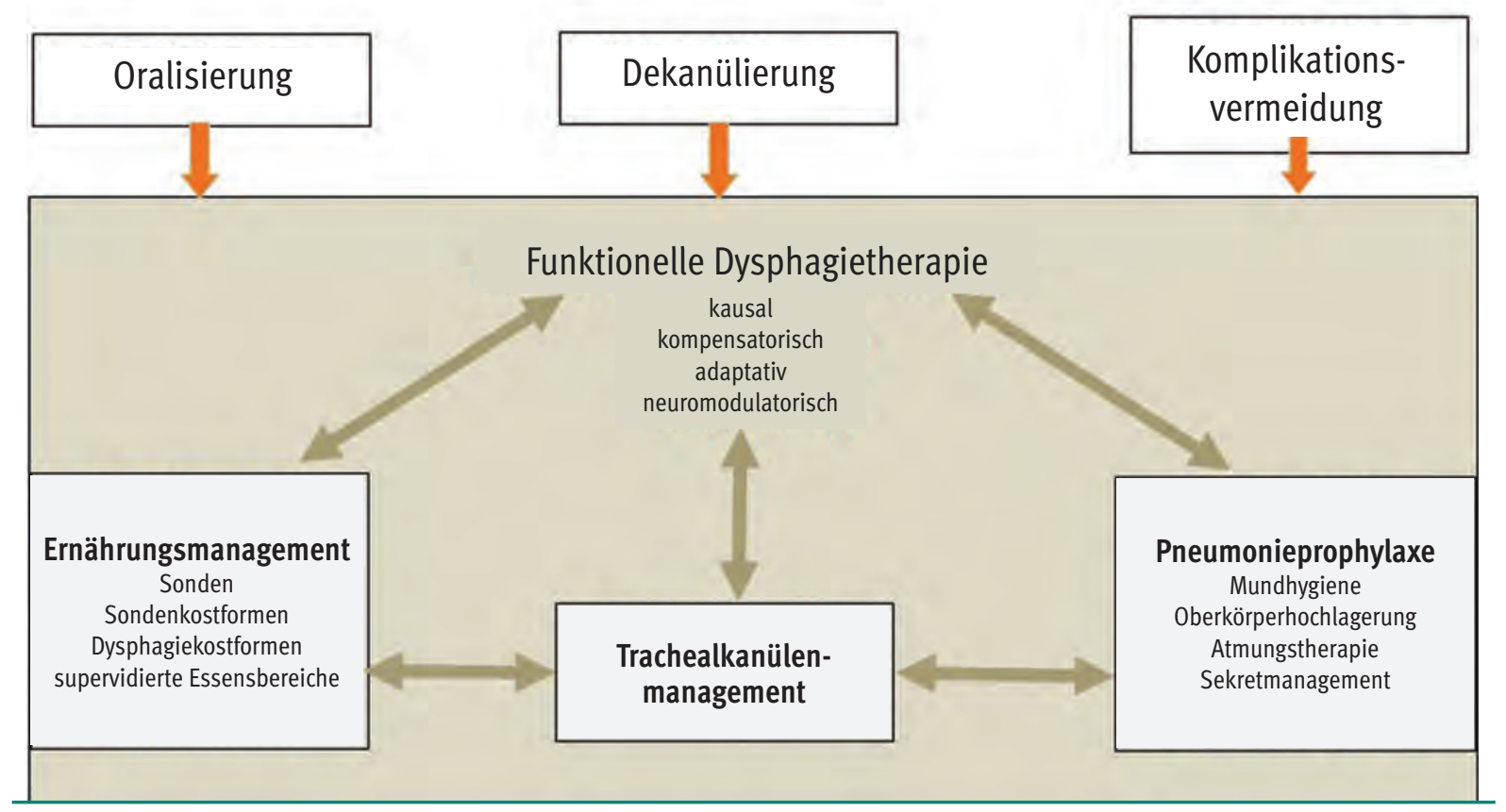

Abb. 10: Dysphagiemanagement: Ziele und Behandlungselemente

Primäres Ziel im dysphagisch-frührehabilitativen Therapieverlauf ist es, eine Deoralisierung des Patienten $\mathrm{zu}$ vermeiden bzw. $\mathrm{zu}$ beenden. Dies geschieht in Abwägung des diagnostisch ermittelten Risikoprofils und unter Anwendung kompensatorischer und adaptativer Therapieverfahren. Das Schlucken selbst geringer Mengen trägt nicht nur entscheidend zur Lebensqualität bei, sondern durchbricht auch einen negativen Kreislauf. Endoskopisch sind bei deoralisierten Patienten häufig eine reduzierte Schluckfrequenz, zäh-borkige Sekretresiduen und pharyngo-laryngeale Ödeme beobachtbar. Letztere sind am ehesten auf den mangelnden Abtransport der Lymphe durch die fehlende pharyngeale Muskelpumpe beim Schlucken zurückzuführen, bedingen eine Einschränkung der Oberflächensensibilität und damit eine weitere Verschlechterung des Schluckakts. Im Untersuchungsverlauf verbessern sich pharyngo-laryngeale Ödeme und Speichelresiduen häufig nach einigen Schlucken. Vor diesem Hintergrund ist die (partielle) Wiederherstellung und Stimulation der Schluckfähigkeit ein Faktor im Rehabilitationsprozess, der wesentlichen Einfluss auf die pharyngo-laryngeale Morphologie, das Sekretmanagement und letztlich auch auf die Dekanülierbarkeit von Patienten hat.

Funktionale Therapieansätze der Dysphagie (kausal, neuromodulativ, kompensatorisch und adaptativ) werden in variablen Kombinationen zur Funktionsverbesserung und Risikoreduzierung bei therapeutischen
Schluckversuchen eingesetzt; ihre Auswahl erfolgt in Abhängigkeit von der Ätiologie, dem Hirnnervenstatus und der Kooperationsfähigkeit der Patienten.

Aufgrund dissoziierter kortikaler Aktivierungsareale von isolierten oro-fazialen Bewegungen im Gegensatz $\mathrm{zu}$ Bewegungen beim Sprechen und Schlucken wird die Effizienz isolierter Bewegungsübungen zur Behandlung supranukleärer Paresen bezweifelt [103]. In der Konsequenz sind Funktionsübungen anzustreben. Tonisierende Maßnahmen bei supranukleären Paresen sollten allenfalls initial bei schlaffer Muskulatur durchgeführt werden und rasch aufgrund der Gefahr der Entwicklung von Hypertonus durch Funktionsübungen ersetzt werden, um zeitliche und räumliche Koordination $\mathrm{zu}$ trainieren. Detonisierende Maßnahmen bei Spastik oder Hypertonus bewirken kurzfristige Tonusveränderungen ohne längerfristigen Transfer und sollten lediglich vorbereitend eingesetzt werden, um günstigere Voraussetzungen für das Schlucktraining zu schaffen. Bei peripheren Paresen sind Kräftigungsübungen und stimulative Verfahren wie Vibration und Elektrotherapie zur Atrophievermeidung indiziert.

Die Kooperationsfähigkeit frührehabilitativer Patienten ist stark unterschiedlich. Auf der einen Seite kann eine wachsende Zahl von Patienten mit schweren Polyneuropathien aufgrund vergleichsweise geringer Aufmerksamkeitseinschränkungen anspruchsvolle schlucktherapeutische Techniken und Manöver erlernen. Dies 
trifft ebenso für Patienten mit Wallenberg-Syndrom nach Hirnstammläsion zu. Auf der anderen Seite sind aktive Übungen mit minimal responsiven Patienten kaum durchführbar. Die Aktivierung des Schluckens gelingt in dieser Patientengruppe am besten durch Funktionstraining mittels gustatorischer und thermaler Stimulation. Solch aktivierende Verfahren dürften die Wirksamkeit passiver Stimulationstechniken übersteigen. Hilfestellungen zur Erleichterung des willkürlichen oralen Transports sind die Applikation von Bolus auf den Zungengrund oder in die dorsalen Wangentaschen sowie die Auswahl stimulativer Materialien (Eis, mit Zitronensaft versetzte Konsistenzen) und leicht transportierbarer Konsistenzen. Kompensatorische Haltungsänderungen können meist bei antriebsgeminderten Patienten angewandt werden. Bei suffizienter pharyngealer Phase kann die sogenannte Kipptechnik (Kopfretroflexion) angewandt werden.

\section{Kausale Therapieverfahren}

Kausale Verfahren werden eingesetzt, um Paresen und sensorische Defizite zu verbessern. Anwendbare Techniken sind Streichungen, Dehnungen, Vibration, Kräftigungsübungen für Lippen, Zunge, Kieferschluss, Pharynx, Stimmlippenschuss und hyoidale Beweglichkeit. Eine Verbesserung des sensorischen Inputs kann durch Durchblutungsförderung (Streichungen, Massage), Veränderung von Bolusvolumen und -viskosität, Geschmacksintensivierung (insbesondere durch Beimischung von Zitronensaft) sowie durch thermale Reize erreicht werden. Prinzipien dieser Verfahren wurden von Clark [17] und Ledl [59] zusammengefasst, umfangreiche Beschreibungen oro-fazialer Übungen haben Bartholome [6] und Castillo-Morales [16] vorgelegt.

\section{Behandlung der Mundöffnungsstörungen}

Mundöffnungsstörungen finden sich relativ häufig bei Patienten mit schweren zentralen bilateralen Läsionen und resultierender Spastik oder Hypertonus der Kieferschließer. In ungünstigen Kombinationen ist der Trismus kombiniert mit Saugautomatismen, sodass durch den Zungenvorstoß und das Einziehen der Unterlippe schwere Bisswunden entstehen können. Häufig kann die Mundöffnung und eine Triggerung des Schluckakts durch Stimulation des »K-Punktes« [49] erreicht werden. Dieser Punkt liegt auf der Innenseite des Unterkiefers seitlich des Arcus palatoglossus und kann nach dessen Passage mit der Spitze des Zeigefingers erreicht werden. Bisweilen kommt es durch Lockerung der Hals-Nackenmuskulatur und anterolaterale Mobilisation des Kopfes (Basisübung nach Castillo-Morales) zu einer Besserung. Sollte über einen längeren Zeitraum keine Kieferöffnung beobachtet werden, wird eine mechanische Dehnung der Kieferschließer empfohlen, um eine Verkürzung der Muskulatur, der Kiefergelenkssehnen und eine Verknöcherung des Gelenks

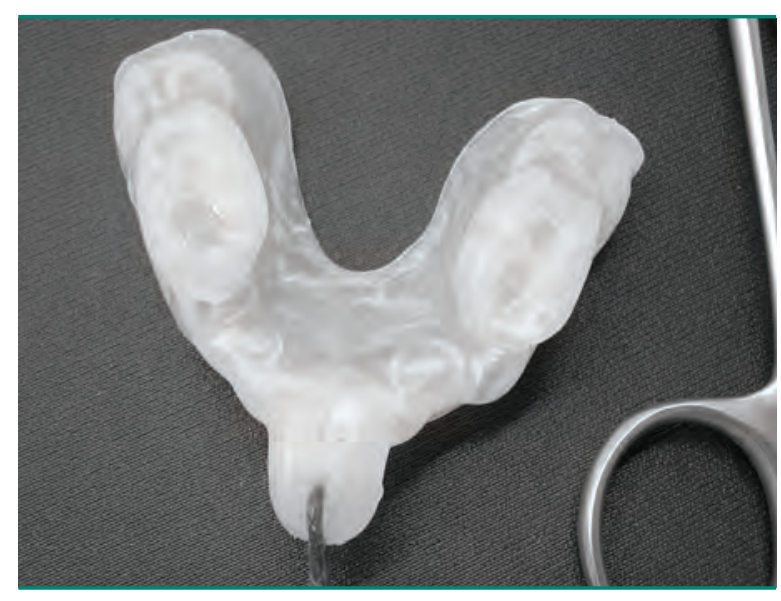

Abb. 11: Aufbissschiene mit Erhöhung an den Molaren zur Therapie bei Mundöffnungsstörungen

zu vermeiden. Dies kann am ehesten über Spateltraining erfolgen. Die Kraftentwicklung mechanischer Kieferöffner (Therabite) ist meist nicht ausreichend. Insbesondere zur längerfristigen Dehnung und bei Gefahr von Bissverletzungen empfiehlt sich der Einsatz von Aufbissschienen, die individuell angefertigt an den Molaren erhöht werden und eine Dauerdehnung bewirken (Abb. 11).

Positive Ergebnisse nach Injektion von Botulinumtoxin in die Kaumuskulatur werden in Fallstudien beschrieben [91], andere betonen die Notwendigkeit randomisierter Studien zur Effizienzbeurteilung der Injektionen bei Trismus [42]. Eine randomisiert kontrollierte Studie an 11 neurologischen Patienten ergab nach Botulinumtoxin-Serotyp-B-Injektionen in die Mm. masseteres eine signifikante Verbesserung der passiven Mundöffnung und von Bisswunden sowie eine Erleichterung der Mundpflege [31]. Unserer Erfahrung nach sollten Injektionen in alle Kieferschließer erfolgen, zumindest aber in die Mm. masseteres und die Mm. temporales, da diese synergetisch arbeiten.

\section{Kompensatorische Verfahren}

Kompensationstechniken umfassen Schluckmanöver, Haltungsänderungen und Erhöhung des sensorischen Inputs. Sie werden bei ungünstiger Prognose als vorrangige Therapiemethodik eingesetzt, finden aber ebenso Anwendung bei günstiger Prognose, dann meist in Kombination mit kausalen Techniken.

Wichtige kompensatorische Manöver sind das Mendelsohn Manöver [46], das supraglottische und das super-supraglottische Schlucken [72], »effortful swallow« [46] und Haltungsänderungen. Letztere benützen Schwerkrafteffekte, um ein Abgleiten des Bolus über die nicht-paretische Seite oder Zeitgewinn beim Schlucken durch Verlangsamung des Bolus zu erreichen. Rasley et al. [78] konnten durch Anwendung kompensatorischer Haltungsänderungen Aspiration bei 77\% der Patienten 
Tab. 2: Indikationen und Effekte kompensatorischer Haltungsänderungen

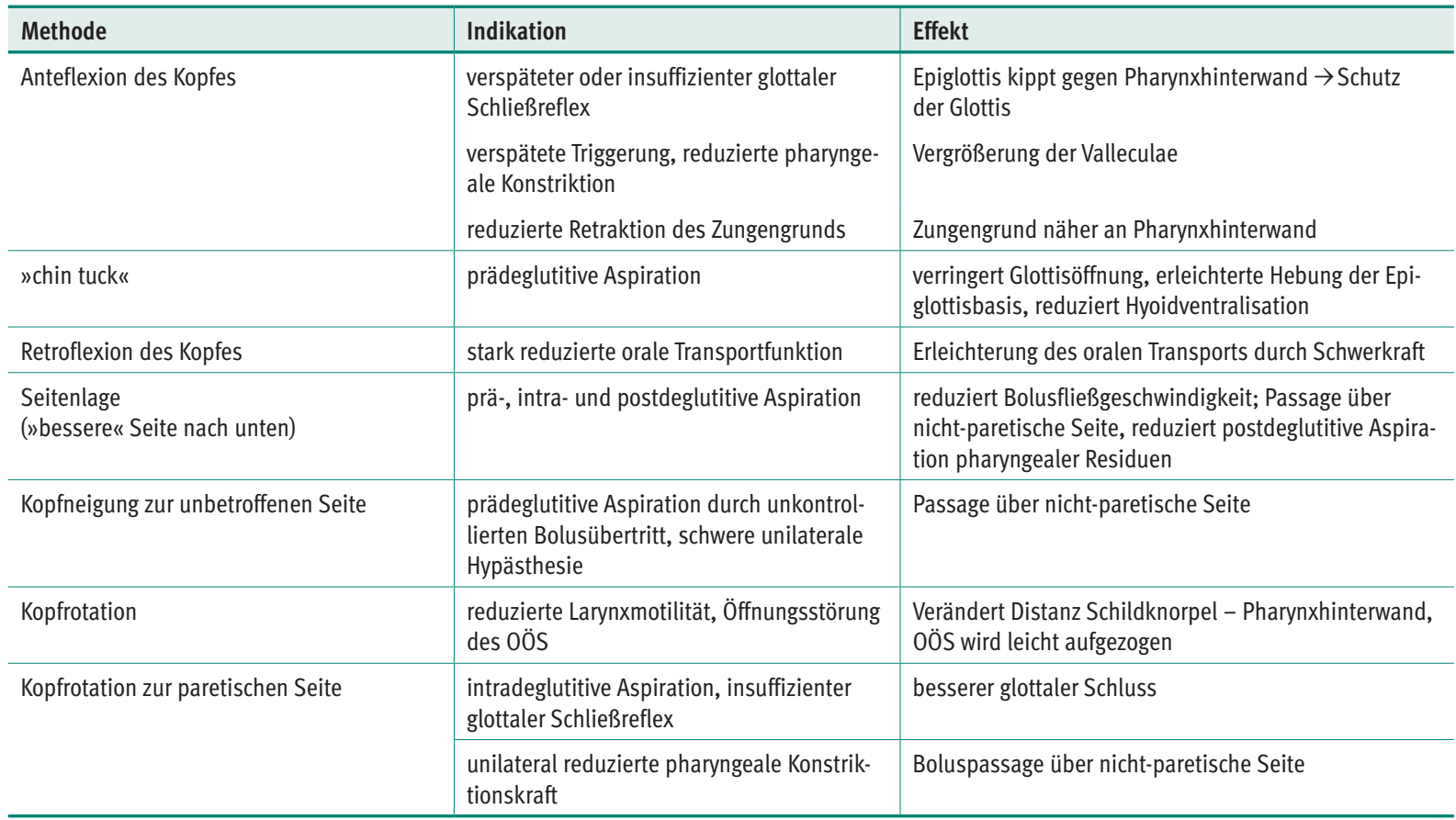

reduzieren und bei $25 \%$ der Patienten gänzlich abwenden. Der Gebrauch und die Anwendungslogik kausaler und kompensatorischer Therapieverfahren unterscheiden sich nicht in der NNFR im Vergleich zu anderen Rehabilitationsphasen. Während kompensatorische Manöver bei nicht oder eingeschränkt kooperationsfähigen Patienten häufig schwer umsetzbar sind, lassen sich Haltungsänderungen (Tab. 2) stets anwenden.

\section{Prothetische und adaptative Maßnahmen}

Bei peripheren Hypoglossus-Paresen kommt es infolge des eingeschränkten Stempeldrucks der Zunge zu oralen Residuen und eingeschränkter Transportfunktion. Durch Verkürzung des Zungenwegs gegen den harten Gaumen kann diese Symptomatik gelindert werden. Dazu wird eine Gaumenprothese angepasst, die einen Teil der Gaumenform auskleidet (Abb. 12).

\section{Adaptative Verfahren}

Nahrungsadaptation ist eines der effektivsten Verfahren zur Vermeidung von Aspiration. Sie erfolgt gemäß den physiologischen Ursachen einer Schluckstörung und wird meist in einem instrumentellen Verfahren ausgetestet. Bei verminderter OÖS-Öffnung werden Flüssigkeiten leichter in den Ösophagus eingeschluckt als feste Konsistenzen. Letztere sind bei Hypästhesie besser spürbar, sodass im Vergleich zu breiigen Konsistenzen eine verbesserte orale Kontrolle resultieren kann. Normotherme Konsistenzen - und dabei insbesondere Speichel - stimulieren intraorale Thermorezeptoren gering,

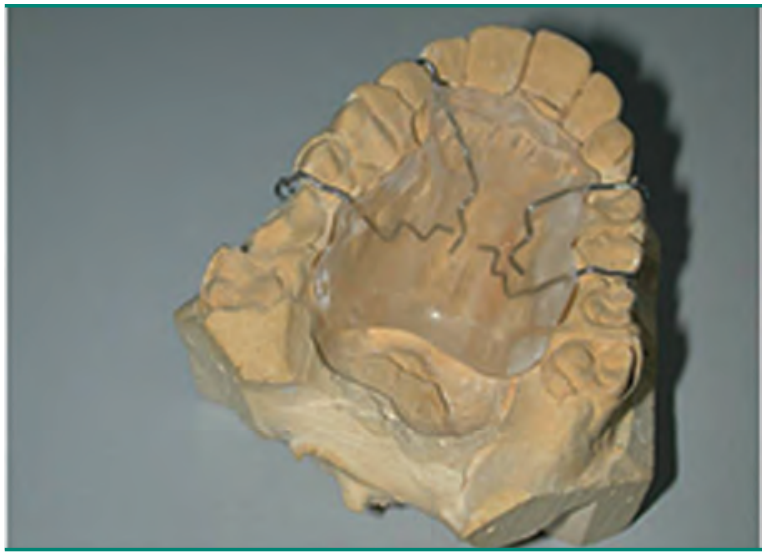

Abb. 12: Gaumenprothese

sodass bei Hypästhesie insbesondere ein Wechsel kalter und warmer Konsistenzen vorteilhaft sein kann. Breiige Konsistenzen empfehlen sich bei eingeschränkter Kaufunktion und reduzierter Zungenkraft. Gemischte oder krümelige Konsistenzen sind schwerer kontrollierbar als einheitliche, von klebrigen Konsistenzen wird abgeraten. Die Fließgeschwindigkeit von Flüssigkeiten kann durch Andicken (verschiedene industrielle Produkte erhältlich) oder durch die Wahl dickflüssiger Säfte verringert werden.

\section{Esshilfen}

Das nicht-selbstständige Essen ist mit einem erhöhten Pneumonierisiko assoziiert. Wichtige Hilfen zum selbst- 
ständigen Essen sind rutschhemmende Unterlagen für Teller, Tellerranderhöhungen, Griffverdickungen für Essbesteck, angepasste Trinkgefäße und Help-Arme zur Gewichtsentlastung paretischer Arme. Trinkbecher mit Dosierfunktion sind eine wichtige Hilfe für ataktische Patienten oder Patienten mit Gaumensegelparesen oder Impulskontrollstörungen. Das Saugen am Schnabelbecher kann bei pathologischem Saugreflex rehabilitative Bemühungen zum Aufbau von Willkürmotorik konterkarieren. Trinken mit Strohhalm erlaubt eine Anteflexion des Kopfes und kann die Flüssigkeitsaufnahme bei insuffizientem dorso-velarem Verschluss erleichtern, ist aber bei Patienten mit Saugautomatismen ebenso kontraindiziert. Letzteren Patienten gelingt es meist nicht, Nahrung vom Löffel abzunehmen, sodass der Gebrauch von Löffeln mit flexibler Schüssel vorteilhaft sein kann. Bei Ausfall der oralen Transportfunktion und bei gleichzeitig ausreichender pharyngealer Phase kann Nahrung unter Umgehung der oralen Phase auf den Zungengrund verabreicht werden. Dies kann z. B. über eine Spritze mit flexiblem Verlängerungsschlauch erfolgen.

\section{Neuromodulative Verfahren}

An dysphagischen Patienten konnte gezeigt werden, dass die Reorganisation des Schluckaktes nach Hemisphärenläsionen durch Aktivierung der nicht-betroffenen Hemisphäre erfolgt [35]. Der Einsatz neuromodulativer Verfahren soll diesen Prozess unterstützen. Sie werden unterschieden in zentrale und periphere Stimulationstechniken.

Zentrale Techniken sind repetitive transkranielle Magnetstimulation (rTMS) und transkranielle Gleichstromstimulation (tDCS). Pisegna et al. [75] konnten für diese Verfahren in einer Metaanalyse einen signifikanten Effekt kortikaler Reorganisation nach Stimulation der nicht-betroffenen Hemisphäre bei Schlaganfallpatienten belegen, betonen allerdings die noch geringe Zahl stimulierter Patienten und die Notwendigkeit höherer Fallzahlen.

Periphere Techniken werden unterteilt nach motorisch-neuromuskulären Verfahren (VitalStim ${ }^{\circledR}$ ), die eine Muskelkontraktion intendieren, und sensorischen Verfahren (pharyngeale Elektrostimulation: PES), bei denen mit niedrigschwelligen Intensitäten stimuliert wird.

\section{VitalStim $^{\circledR}$}

VitalStim ${ }^{\circledR}$ intendiert eine Verbesserung des Schluckakts durch elektrische Stimulation der hyoidalen Muskulatur. Es werden Oberflächenelektroden oberhalb des Larynx platziert, die Intensität der transkutanen Stimulation wird so lange gesteigert, bis es zur Kontraktion der relevanten tieferliegenden Muskulatur (M. thyrohyoideus, M. mylohyoideus) kommt. Doeltgen et al. [25] betonten den Einfluss von Stimulationsfrequenz, -latenz und Dosis auf die kortikale Aktivierung bei Gesunden.
Die vorliegenden Studienergebnisse $\mathrm{zu}$ VitalStim ${ }^{\circledR}$ sind widersprüchlich. Positive Therapieeffekte wurden initial von Freed et al. [33] berichtet, deren Ergebnismessung allerdings mittels nicht validierter Skalen erfolgt war. Keine Vorteile von VitalStim ${ }^{\circledR}$-Therapie gegenüber traditioneller Schlucktherapie ergaben sich bei Bülow et al. [11] und Kiger et al. [48].

Ludlow et al. [62] beobachteten als negativen Effekt eine Depression des Hyoids bei Normalpersonen und wiesen damit auf eine Verschlechterung des Schluckakts und auf potenzielle Risiken dieses Verfahrens hin.

In der Zusammenschau gibt es für VitalStim ${ }^{\circledR}$ Therapie keine gesicherten Angaben zu Häufigkeit, Dauer, Intensität und Frequenz der Stimulation. Auch wenn mehrere Studien Behandlungsvorteile in Kombination mit traditioneller Schlucktherapie berichten, ist der therapeutische Nutzen des Verfahrens nicht ausreichend gesichert.

\section{Pharyngeale Elektrostimulation (PES)}

Zur pharyngealen Stimulation wird eine bipolare Elektrode in einer flexiblen Sonde transnasal bis nach laryngeal vorgeschoben. Im Bereich der retrolaryngealen Pharynxhinterwand erfolgt eine sensorische Stimulation unterhalb der Schmerzschwelle (Abb. 13). Fraser et al. [32] wiesen eine Veränderung der kortikalen Topographie schluckrelevanter Areale in Abhängigkeit von Frequenz, Dauer und Intensität der pharyngealen Stimulation nach. Der Effekt baute sich 60-90 Minuten nach Stimulation auf und korrelierte mit einer signifikanten Verbesserung der Aspirationsgrade dysphagischer Patienten. Eine Veränderung der Hirnstammaktivität konnte nicht nachgewiesen werden. Nach pharyngealer Stimulation bihemisphärisch läsionierter Patienten fand Ledl [58] keine persistierende Verbesserung der Schluckfrequenz oder der Aspirationsgrade im Vergleich zur Kontrollgruppe, sodass der Effekt der PES nur nach monohemisphärischen Läsionen durch Aktivierung der nicht-betroffenen Hemisphäre entsteht. Jayasekeran et

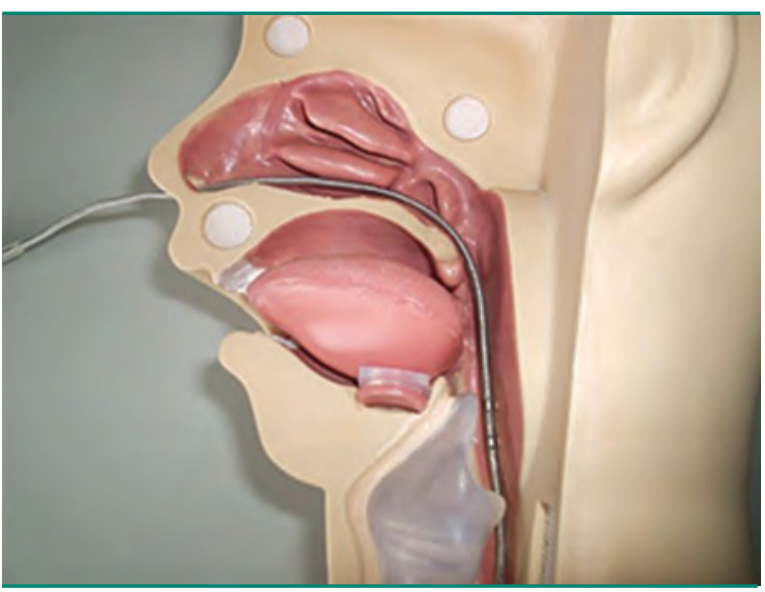

Abb. 13: Pharyngeale Elektrostimulation 
al. [43] wiesen eine Umkehrung virtueller kortikaler Läsionen durch rTMS mittels PES nach und fanden in einer kontrolliert-randomisierten klinischen Studie einen Rückgang von Aspiration und Krankenhausaufenthaltsdauer in der experimentellen Gruppe. Suntrup et al. [90] konnten in einer kontrolliert randomisierten Studie an tracheotomierten Patienten eine signifikant höhere Dekanülierungsrate der stimulierten Gruppe nachweisen, Restivo et al. [79] einen verbesserten Dysphagie-Outcome bei Patienten mit Multipler Sklerose. Eine multizentrische randomisiert kontrollierte Studie [7] konnte zwar die Sicherheit des Verfahrens belegen, aber keine Verbesserung des Outcomes durch die PES. Damit kann die PES derzeit nur für die Behandlung selektiver Patientengruppen und Ätiologien empfohlen werden. Angesichts zahlreicher positiver Proof-ofPrinciple-Studien ist $\mathrm{zu}$ hoffen, dass zur weiteren Verbesserung des Behandlungsoutcomes in naher Zukunft neuromodulative Verfahren als adjuvante Therapietechniken zur Verfügung stehen werden.

\section{Pneumonieprophylaxe}

Die Aspirationspneumonie ist die häufigste Form der nosokomialen Pneumonie und zeigt eine Mortalität von $20-65 \%$. Infektionen waren in einer prospektiven Kohortenstudie über 2.130 Schädel-Hirn-Verletzte in der stationären Rehabilitation mit über $25 \%$ auch der häufigste Grund für die Rückverlegung in die akutstationäre Behandlung. Dabei stellten sich die (Aspirations-) Pneumonien mit knapp 46\% mit weitem Abstand als die häufigste Ursache heraus [36]. Als geeignete Maßnahmen der Pneumonieprophylaxe werden die hygienische Mundpflege, die Oberkörperhochlagerung, die Atmungstherapie und das medikamentöse Sekretmanagement diskutiert.

\section{Hygienische Mundpflege}

Als Risikofaktoren für eine Aspirationspneumonie gelten höheres Lebensalter, Mangelernährung mit resultierender Immunschwäche, Rauchen, schlechte Mundhygiene, antibiotische Vorbehandlung, Xerostomie und orale Nahrungskarenz. Diese prädisponieren für eine Überwucherung der gesunden oralen Flora mit pathogenen, v.a. aeroben gramnegativen Bakterien (AGNB), die bei Gesunden nicht oral nachweisbar sind [8o]. Bei Schlaganfallpatienten ist sowohl in der Akutbehandlung als auch in der Rehabilitation die orale Kolonisation mit AGNB nachgewiesen, wobei diese mit der Schwere der Erkrankung korreliert: $37 \%$ der moderat und $73 \%$ der schwer Erkrankten weisen eine orale Kolonisation mit AGNB auf, die bei der Aspirationspneumonie am häufigsten als pathogene Erreger isoliert werden [34].

Die regelmäßige Mundpflege mit mechanischer Zahnreinigung ist eine wichtige Maßnahme der Grund- pflege. Ob sie allerdings auch einen Beitrag zur Pneumonieprophylaxe leistet, ist noch nicht hinreichend belegt. Metaanalysen mit kleiner Patientenzahl und hoher Heterogenität konnten keinen Vorteil der mechanischen Zahnreinigung nachweisen [50].

Demgegenüber führt antiseptische Mundspülung mit Chlorhexidin bei kardiochirurgischen Intensivpatienten zu einer reduzierten Pneumonieinzidenz. Gab es zunächst widersprüchliche Daten hierzu, konnte in mehreren Metaanalysen ein protektiver Effekt von Chlorhexidin Mundspülung für beatmungsassoziierte Pneumonien nachgewiesen werden. Allerdings wird für die Anwendung von Chlorhexidin eine Erregerverschiebung gerade in den gramnegativen Bereich befürchtet, da Chlorhexidin hier schlechter wirksam ist als im grampositiven Spektrum [50].

Auch wenn eine Studie zur selektiven oralen Dekontamination bei über 200 Schlaganfallpatienten durch die orale Applikation von Colistin, Amphotericin B und Polymyxin E als Gel die Kolonisation mit gramnegativen Bakterien und die Pneumonierate signifikant reduzierte [34], konnte ein Effekt auf Letalität oder Sepsisrate durch die Anwendung einer oralen antiseptischen Dekontamination bisher nicht dargestellt werden. Auch wird diesbezüglich das Risiko der Entwicklung weiterer Multiresistenzen befürchtet [50].

\section{Oberkörperhochlagerung}

Die Oberkörperhochlagerung zur Prophylaxe der nosokomialen (Aspirations-)Pneumonie ist fester Bestandteil vieler Empfehlungen. So wird für die stationäre und ambulante Pflege eine Oberkörperhochlagerung für bis zu 30 Minuten nach oraler oder enteraler Nahrungsaufnahme empfohlen. Bei einem Vergleich einer Oberkörperhochlagerung von $28^{\circ}$ gegenüber $10^{\circ}$ ergab sich kein Unterschied in der Inzidenz der beatmungsassoziierten Pneumonie, sodass eine Oberkörperhochlagerung $>30^{\circ}$ nicht generell empfohlen wird, da es außerhalb sogenannter Präventionsbündel keine Evidenz für eine Oberkörperhochlagerung von beatmeten Patienten zur Senkung der Pneumonierate gebe [50].

\section{Atmungstherapie bei Dysphagiepatienten}

Sind neurologisch-neurochirurgische Patienten bereits aufgrund ihrer neuromuskulären Einschränkung mit negativer Auswirkung auf Mobilität, Sekretreinigung und die Atempumpe häufig auf regelmäßige Atmungstherapie angewiesen, so sind sie es umso mehr bei Vorliegen einer Dysphagie mit begleitenden Aspirationsepisoden. Im Unterschied zu pulmologischen Patienten erschweren Einschränkungen in der Vigilanz, Kognition, Wahrnehmung, Aufmerksamkeitsspanne und Kooperationsfähigkeit die Therapie. 
Tab. 3: Maschinelle Atemtherapie: Welche Therapie mit welcher Wirkung bei welchem Patienten?

\begin{tabular}{|c|c|c|c|c|c|c|}
\hline Atemtherapie & $\begin{array}{l}\text { Incentive Spiro- } \\
\text { meter }\end{array}$ & $\begin{array}{l}\text { PEP-System - oszil- } \\
\text { lierend }\end{array}$ & $\begin{array}{l}\text { PEP-System - nicht } \\
\text { oszillierend: EzPAP }\end{array}$ & $\begin{array}{l}\text { Bagging/ } \\
\text { Air Stacking }\end{array}$ & IPPV-Gerät & $\begin{array}{l}\text { Mechanischer In- } \\
\text { und Exsufflator }\end{array}$ \\
\hline Wirkweise & aktiv & aktiv & teilaktiv & passiv & passiv & passiv \\
\hline Wirkung & Muskelaufbau & $\begin{array}{l}\text { Stabilisierung der } \\
\text { Bronchien, } \\
\text { Sekretmobilisation }\end{array}$ & $\begin{array}{l}\text { Muskelaufbau und } \\
\text { Blähen }\end{array}$ & $\begin{array}{l}\text { Blähen und } \\
\text { Sekretolyse }\end{array}$ & Blähen & $\begin{array}{l}\text { Blähen und Sau- } \\
\text { gen } \\
\text { Sekretolyse }\end{array}$ \\
\hline $\begin{array}{l}\text { kooperativer } \\
\text { Patient }\end{array}$ & ja & ja & ja & ja & ja & ja \\
\hline $\begin{array}{l}\text { nicht kooperativ/ } \\
\text { komatös }\end{array}$ & nein & nein & ja & ja & ja & ja \\
\hline $\begin{array}{l}\text { hoher QS/ } \\
\text { Tetraplegie }\end{array}$ & nein & nein & nein/ja & ja & ja & ja \\
\hline Paraplegie & ja & ja & ja & ja & ja & ja \\
\hline $\begin{array}{l}\text { Reduzierte Atem- } \\
\text { arbeit/ } \\
\text { Erschöpfung }\end{array}$ & nein & nein & nein & ja & ja & ja \\
\hline $\begin{array}{l}\text { Dystelektasen } \\
\text { Atelektasen } \\
\text { Sekretstau }\end{array}$ & ja & ja & ja & ja & ja & ja \\
\hline
\end{tabular}

Die Indikationsstellung zur Atmungstherapie ergibt sich aus den Funktionsparametern der Atmung:

- Sauerstoffsättigung < $92 \%$ bzw. Sauerstoffbedarf für eine stabile $\mathrm{SpO}_{2}>92 \%$

- $\mathrm{PaCO}_{2} \geq 50 \mathrm{mmHg}$

- Atemfrequenz $>25 \mathrm{~b} / \mathrm{min}$

- Hustenstoß: PCF (peak cough flow) <270l/min, alternativ Reduktion von Vitalkapazität oder Atemzugvolumen um $30 \%$

\section{Risikofaktoren und Begleiterkrankungen:}

- Erkrankung der Lunge (Pneumonie, COPD)

- Reduzierte Atempumpe (bei CIP/CIM, GBS, Myasthenie, hoher Querschnittslähmung)

- schwere Dysphagie

Die wichtigsten Inhalte und Ziele der Atemtherapie sind:

- physiotherapeutische und physikalische Maßnahmen zur

- Verbesserung der Beweglichkeit, Steigerung der Thoraxexkursion

- Kräftigung der Atem- und Atemhilfsmuskulatur [82]

- Mobilisation zur Verbesserung der Rumpfkontrolle

- Erhöhung des Tidalvolumens: führt zu effektiverem Husten, vergrößert die mechanische Compliance, reduziert Atemarbeit, Dys- und Atelektasen. Maßnahmen: aktives inspiratorisches Training (incentive Spirometer), passive Inspirationstherapie mit IPPVGeräten (intermittent positive pressure ventilation)

- Verbesserung des Hustenstoßes und der Sekretmobilisierung durch:
- Lagerung/Positionierung des Patienten [9]

- Erhöhung des intrapulmonalen Volumens und Rekrutierung minderbelüfteter Alveolen, z.B. durch Luft stapeln (air stacking) [4], manuelle Hyperinflation (Bagging), IPPV-Geräte

- Erhöhung des Exspirationsflusses beim Husten durch assistierte Hustentechniken oder Einsatz von mechanischen Hustenhilfen (mechanische In- und Exsufflatoren),

- Eröffnung von Dystelektasen und Sekretmobilisation durch Anwendung von oszillierenden oder nicht oszillierenden PEP-Geräten $(\mathrm{PEP}=$ positive expiratory pressure).

Bis auf die oszillierenden PEP-Geräte können alle maschinellen Atemtherapiegeräte über Mundstück, Maske oder Trachealkanüle angewendet werden. Die Verwendung eines incentive Spirometers via Trachealkanüle ist nur möglich mit Gerätemodellen, die auch die Exspiration über das Gerät erlauben. Wenn das mobilisierte Sekret nicht über die Trachealkanüle abgehustet werden kann, muss es unter Beachtung der Hygienevorschriften abgesaugt werden.

Bedauerlicherweise fehlen bislang klinische Studien sowohl bezüglich der Effektivität als auch der Anwendungshäufigkeit von Atmungstherapie bei neurologischneurochirurgischen Rehabilitationspatienten. Dennoch haben sich klare Vorgaben und Stratifizierungen (wer bekommt wann, was, warum?) im klinischen Alltag der neurologischen Frührehabilitation bewährt (siehe Tab. 3).

Die Durchführung einer individuellen, komplexen Atmungstherapie gelingt nur im Team, idealerweise unter der Leitung und Supervision eines Atmungsthera- 
peuten. Dieser sollte sowohl die Nachhaltigkeit als auch die Effektivität der durchgeführten Therapien im Auge haben.

\section{Medikamentöses Sekretmanagement}

Sialorrhoe bei Patienten der NNFR ist in aller Regel Resultat einer Schluckstörung [70]. Diese kann durch eine Vielzahl an Medikamenten aggraviert werden (z.B. Neurolepetika, Sedativa), selten liegen eine unmittelbare (toxische) medikamentöse Nebenwirkung (z.B. Cholinergika) oder orale, pharyngeale oder ösophageale entzündliche Veränderungen zugrunde. In der NNFR sollte deshalb unseres Erachtens im Gegensatz zur S2kLeitlinie Hypersalivation [24] der Terminus Sialorrhoe bevorzugt werden. Zur Vermeidung der Sialorrhoe sind ungestörte neuronale und muskuläre Abläufe insbesondere oro-pharyngeal sowie eine ausreichende Schluckfrequenz erforderlich. Primäres Ziel in der Behandlung der Sialorrhoe muss eine Verbesserung der Schluckstörung und ein Vermeiden von aggravierenden Faktoren sein.

Die meist sonographisch kontrollierte Injektion von Botulinumtoxin (BTX) A und B in die großen Speicheldrüsen (Gl. parotis und Gl. submandibularis) erscheint gegenwärtig aufgrund des Grades der Evidenz (Level B bzw. Level A nach AAN-Leitlinien) und der niedrigen Komplikationsrate bei Injektion durch einen erfahrenen Behandler als die effektivste Behandlung der Sialorrhoe bei einer ganzen Reihe neurologischer Erkrankungen, wobei sich Effekt und Nebenwirkungsspektrum bei beiden Toxinen nicht wesentlich unterscheiden [52]. Sie kann für ein breites Alters- und Krankheitsspektrum empfohlen werden. Es ist jedoch darauf hinzuweisen, dass keines der BTX für diese Indikation zugelassen ist und dementsprechend eine besondere Aufklärungspflicht besteht.

Demgegenüber ist eine symptomatische medikamentöse Behandlung häufig nur begrenzt wirksam, sodass die oralen Substanzen nur als »wahrscheinlich wirksam« eingeschätzt werden [52]. In einer kleinen doppelblinden, placebokontrollierten Studie zeigte sich für Glykopyrrolat nur bei knapp 40\% der untersuchten Parkinson-Patienten ein klinisch relevanter Effekt einer Speichelreduktion von mind. 30\%. Zudem muss auf das insbesondere bei älteren Patienten doch erhebliche Nebenwirkungsspektrum hingewiesen werden. Auch sind die meisten der unten aufgeführten Medikamente für die Behandlung der Sialorrhoe nicht zugelassen und können ebenfalls nur off-label im Rahmen eines individuellen Heilversuches eingesetzt werden, falls die Grunderkrankung oder Komorbiditäten dies zulassen. Hierzu gehören v.a. oral angewandte Anticholinergika (Muskarinrezeptor-Antagonisten) wie Atropin (oral, sublingual, subkutan), Scopolamin (transdermal, enteral als wässrige Lösung, inhalativ), Ipratropium-Bromid (als
Spray sublingual), Gylcopyrrolat (zugelassen intravenös und intramuskulär; in den USA auch für die orale Gabe bei Kindern), Trihexiphenidylbenzhexol-Hydrochlorid (oral/enteral), Pirenzepin und Amitriptylin (oral/enteral) [24]. Sinnvolle medikamentöse Therapien bei akuter Hypersalivation sind insbesondere die verschiedenen Applikationsformen von Glycopyrrolat und Scopolamin, jedoch ist die Langzeitanwendung kritisch $\mathrm{zu}$ betrachten.

Als Ultima Ratio können chirurgische Speicheldrüseneingriffe wie die Submandibulektomie, die Photokoagulation der Speicheldrüsengänge oder eine externe Bestrahlung in Erwägung gezogen werden [24, 70].

\section{Verbesserung der Tracheo-Bronchialtoilette}

Der fehlenden bzw. eingeschränkten tracheobronchialen Reinigungsfunktion durch Störung des Hustenreflexes kommt bei der Entstehung einer Aspirationspneumonie eine entscheidende Bedeutung zu (stille Aspiration oder "silent aspiration«). Bei gebrechlichen älteren Menschen (»frailty«) kommt es über eine physiologische Presbyphagie häufig zu einer chronischen Mikroaspiration und durch die hierdurch induzierte Zytokinausschüttung zu kortikalen und bulbären neuralen Störungen des Hustenreflexes (»dystussia»), aber auch zu einer Sarkopenie mit unmittelbarer Schwächung der motorischen Reinigungsfunktionen [28].

Neben pflegerischen Maßnahmen zum Sekretmanagement mit Sekretolyse und Sekretmobilisation, Sekretelimination und den oben beschriebenen Möglichkeiten der maschinellen Atmungstherapie können auch Medikamente (off-label) zum Einsatz kommen, wobei sich eine starke Empfehlung mangels hinreichender Evidenz in der Literatur nicht findet.

Zur Verbesserung der tracheobronchialen Reinigungsfunktion werden meist Mukolytika eingesetzt, um das Trachealsekret ausreichend flüssig zu halten, damit es gut abgehustet oder abgesaugt werden kann. Auf eine ausreichende Flüssigkeitszufuhr ist dabei zu achten.

Für den Einsatz von ACE-Hemmern legt die Studienlage bei akuten und post-akuten Schlaganfallpatienten einen positiven Effekt hinsichtlich der Verbesserung der Schluckstörung und des Hustenreflexes sowie der Prophylaxe einer Aspirationspneumonie nahe [14]. Pharmakologisch wird als Wirkmechanismus die Hemmung des Abbaus von Substanz P und ein verbesserter Hustenstoß angenommen. Auch für Dopaminergika (L-DOPA, Amantadin) ist eine Verbesserung von Schluckstörung und Hustenfunktion beschrieben [65, 104]. Amantadin wird im Gegensatz zu den ACE-Hemmern in der aktuellen Leitlinie »Neurogene Dysphagien« der Deutschen Gesellschaft für Neurologie zur Prophylaxe einer Aspirationspneumonie bei dysphagischen Schlaganfallpatienten empfohlen [23].

Nicht selten besteht bei Patienten mit Trachealkanüle eine tracheobronchiale Hypersekretion, die aus 
theoretischen Überlegungen mit den bei der Sialorrhoe genannten systemisch wirksamen Anticholinergika (v.a. Glycopyrrolat), insbesondere aber mit Ipratropium-Bromid als Aerosol, behandelt werden kann. Eine Evidenz hierzu gibt es nicht, der Einsatz ist auch hier off-label.

\section{Mangelernährung und Ernährungsmanagement}

Die meisten Patienten der NNFR leiden an einer schweren Grunderkrankung (Schlaganfall, Schädel-Hirn-Trauma, große Operation oder Intensivaufenthalt) und sind nicht in der Lage, die erforderliche Nahrungszufuhr vollständig oral decken zu können (z. B. aufgrund einer Schluckstörung). Zudem zeigen viele einen relevanten Gewichtsverlust im Akutverlauf oder ein Alter über 70 Jahre, sodass man davon ausgehen kann, dass nach der Definition des Nutrition Risk Screenings 2002 [51] fast alle Patienten der NNFR ein hohes Risiko einer Unter-/Mangelernährung aufweisen und damit einer Ernährungsintervention (z.B. Texturmodifikation, orale Supplementierung, enterale Sondenernährung, parenterale Ernährung) bedürfen. Für die Prävalenz der Unter-/ Mangelernährung finden sich auch in Abhängigkeit von der Fachrichtung bereits bei Aufnahme in die Klinik Zahlen zwischen 20 und 60\%. Die Inzidenz der Mangelernährung beträgt bei chronisch kritisch Kranken 43\% [84]. Junge Schädel-Hirn-Verletzte, bei denen eine vorbestehende Unter-/Mangelernährung eher selten sein wird, werden in der Phase der Frührehabilitation zu 60\% als untergewichtig beschrieben [99]. Während der stationären Behandlung im Krankenhaus erleben 30\% bis über $80 \%$ der Patienten einen relevanten Gewichtsverlust.

Folgen einer Mangelernährung sind u.a. die Abnahme der Immunkompetenz mit einer Zunahme der Infektionsrate, -dauer und -schwere, Wundheilungsstörungen und Dekubitus, Zunahme von Immobilität, Pflegebedürftigkeit und Gebrechlichkeit (frailty) und damit letztlich eine Zunahme von Morbidität und Mortalität [61].

Ziel des Ernährungsmanagements auf der Intensivstation wie in der NNFR ist es, einen möglichst großen Anteil der Patienten frühestmöglich adäquat, d. h. ihrem metabolischen Bedarf entsprechend zu ernähren, und damit einen Beitrag zur Reduktion von Morbidität und Mortalität und zur Verbesserung des funktionellen Outcomes zu leisten.

Hierzu geeignete Maßnahmen sind:

- Erfassung des Mangelernährungsrisikos bei allen Patienten z.B. mit Nutritional Risk Screening/NRS 2002 oder NUTRIC-Score (Patienten mit einem hohen Risiko profitieren am meisten von einer Ernährungstherapie).

- Bestimmung des Energiebedarfs bei allen Patienten (orientierend: $25 \mathrm{kcal} / \mathrm{kg}$ )

- Überprüfung des metabolischen Bedarfs im Behandlungsverlauf, regelmäßiges Wiegen, Kalorienbilanzierung mit Überprüfung des Erreichens des Kalo- rienziels unter Verwendung geeigneter Protokolle (z.B. Tellerdiagramm bei oral Ernährten)

- Beginn einer enteralen Ernährung innerhalb von 24-48 Stunden nach Aufnahme mit Deckung von mind. $>80 \%$ des Bedarfs innerhalb von 72 Stunden (Reduktion infektiöser Komplikationen)

- Bei kritisch Kranken, auch Schädel-Hirn-Verletzten, Deckung des erhöhten Proteinbedarfs (1,2-2,o g/kg); auch in der Phase der Rehabilitation scheint noch ein relevanter Proteinkatabolismus zu bestehen

- Adipöse Intensivpatienten sollen hypokalorisch, aber mit hohem Proteingehalt ernährt werden $(2 \mathrm{~g}$ Protein per kg Idealgewicht) (Reduktion Verweildauer auf Intensivstation, aber nicht im Krankenhaus)

- Engmaschige Überprüfung der gastrointestinalen und metabolischen Verträglichkeit der enteralen Ernährung und ggf. kontinuierliche Applikation

- Platzierung der Ernährungssonde jejunal (Reduktion der Rate von beatmungsassoziierter Pneumonie) bei Patienten mit hohem Aspirationsrisiko und/oder gestörter gastrointestinaler Motilität

- Bei Notwendigkeit einer enteralen Ernährung über einen längeren Zeitraum als vier Wochen sollte eine perkutane endoskopische Gastrostomie (PEG) angelegt werden.

In der Literatur finden sich eine Vielzahl aktueller Empfehlungen und Leitlinien zur Ernährung von Patienten mit Schlaganfall, Schädel-Hirnverletzungen und (chronisch) kritischer Erkrankung, auf die hier verwiesen sei [69, 1, 100, 30, 41, 68, 84].

In der NNFR sind spezielle Strukturen und klinikinterne Abläufe zum Ernährungsmanagement sinnvoll. Hierzu gehören das Vorhalten von verschiedenen Präparaten der enteralen Sondenkost und mehrerer oraler Dysphagiekostformen sowie die Schaffung supervidierter Essensbereiche für Patienten mit beaufsichtigungspflichtigen Schluckstörungen.

\section{Literatur}

1. Academy of Nutrition and Dietetics. Critical illness evidence-based nutrition practice guideline. Chicago (IL): Academy of Nutrition and Dietetics, 2012. www.guideline. gov/content.aspx?id=39404; download 10.6.2016.

2. Afkari S. Measuring frequency of spontaneous swallowing. Australas Phys Eng Sci Med 2007; 30(4): 313-317.

3. Aviv JE, Murry T, Zschommler A, Cohen M, Gartner C. Flexible Endoscopic Evaluation of Swallowing With Sensory Testing: Patient Characteristics and Analysis of Safety in 1340 Consecutive Patients. Ann Otol Rhinol Laryngol 2005; 114(3): $173-176$.

4. Bach $\mathrm{J}$ et al. Lung inflation by glossopharyngeal breathing and 'air stacking' in Duchenne muscular dystrophy. Am J Phys Med Rehabil 86(4): S295-S300.

5. Barker $J$ et al. Incidence and impact of dysphagia in patients receiving prolonged endotracheal intubation after cardiac surgrey. Can J Surg 2009; 52(2): 119-24.

6. Bartholome G, Schröter-Morasch H (Hrsg). Schluckstörungen: Diagnostik und Rehabilitation. München: Urban \& Fischer 2010. 
7. Bath P, Scutt P, Love J, Clavé P, Cohen D, Dziewas R, Iversen $\mathrm{H}$, Ledl C, Ragab S, Soda $\mathrm{H}$, Warusevitane A, Woisard V, Hamdy S. Pharyngeal Electrical Stimulation for Treatment of Dysphagia in Subacute Stroke. Stroke 2016; 47: 1562-70.

8. Brady SL, Hildner CD, Hutchins BF. Simultaneous Videofluoroscopic Swallow Study and Modified Evans Blue Dye Procedure: An Evaluation of Blue Dye Visualization in Cases of Known Aspiration. Dysphagia 1999; 14(3): 150-1.

9. Branson RD. Secretion management in the mechanically ventilated patient. Respir Care, 2007 Oct; 52(10): 1328-42.

10. Brogan E, Langdon C, Brookes K, Budgeon C, Blacker D. Respiratory Infections in Acute Stroke: Nasogastric tubes and Immmobility are Stronger Predictors than Dysphagia. Dysphagia 2014; 29(3): 340-5.

11. Bülow M, Speyer R, Baijens L, Woisard V, Ekberg O. Neuromuscular Electrical Stimulation (NMES) in Stroke Patients with Oral and Pharyngeal Dysfunction. Dysphagia 2008; 23: 302-9.

12. Bundesarbeitsgemeinschaft für Rehabilitation BAR (Herausgeber). Empfehlungen zur Neurologischen Rehabilitation von Patienten mit schweren und schwersten Hirnschädigungen in den Phasen B und C, 2. November 1995. Frankfurt am Main, Ausgabe 1999.

13. Butler SG, Stuart A, Castell D, Russell GB, Koch K, Kemp S. Effects of age, gender, bolus condition, viscosity, and volume on pharyngeal and upper esophageal sphincter pressure and temporal measurements during swallowing. JSHLR 2009; 52(1): 240-53.

14. Caldeira D, Alarcao J, et al. Risk of pneumonia associated with use of angiotensin converting enzyme inhibitors and angiotensin receptor blockers: systematic review and meta-analysis. BMJ 2012; 345: e4260.

15. Cameron JL, Reynolds J, Zuidema GD. Aspiration in Patients With Tracheostomies. Surg Gynecol Obstet 1973; 136(1): 68-70

16. Castillo Morales R. Die orofaziale Regulationstherapie. München: Pflaum Verlag 1991.

17. Clark HM. Neuromuscular Treatments for Speech and Swallowing: A Tutorial. Am J Speech Lang Pathol 2003; 12(4): 400-15.

18. Colodny N. Interjudge and intrajudge reliabilities in fiberoptic endoscopic evaluation of swallowing (fees) using the penetration-aspiration scale: a replication study. Dysphagia 2002; 17: 308-15.

19. Cvejic L, Harding R, Churchward T, Turton A, Finlay P, Massey D, Bardin P, Guy P. Laryngeal Penetration and Aspiration in Individuals with Stable COPD. Respirology 2011; 16: 269-75.

20. Daniels S, McAdam C, Brailey K, Foundas A. Clinical Assessment of Swallowing and prediction of dysphagia severity. Am J Speech Lang Pathol 1997; 6: 17-24.

21. Daniels S, Pathak S, Stach C, Mohr T, Morgan R. Speech Pathology Reliability for Stroke Swallowing Screening Items. Dysphagia 2015; 30: 565-570.

22. Davies AE, Kidd D, Stone SP, MacMahon J. Pharyngeal sensation and gag reflex in healthy subjects. Lancet 1995; 345(8948): 487-8.

23. Deutsche Gesellschaft für Neurologie. Neurogene Dysphagien. S1-Leitlinie, Stand 09/2012. www.dgn.org/images/ red_leitlinien/LL_2014/PDFs_Download/030111_DGN_LL_ neurogene_dysphagien_final.pdf. download 13.06.2016.

24. Deutsche Gesellschaft für Hals-Nasen-Ohrenheilkunde, Kopf- und Halschirurgie. Hypersalivation. S2k-Leitlinie, Stand 01/2013. AWMF. www.awmf.org/leitlinien/detail/ 1l/o17-075.html. download 13.6.2016.

25. Doeltgen S, Dalrymple-Alford J, Ridding M, Huckabee M. Differential Effects of Neuromuscular Stimulation. Parameters on Submental Motor-Evoked Potentials. Neurorehabil Neural Repair 2010; 24(6): 519-27.
26. Donzelli J, Brady S, Wesling M, Craney M. Simultaneous modified Evans blue dye procedure and video nasal endoscopic evaluation of the swallow. Laryngoscope 2001; 111(10): $1746-50$.

27. Dziewas R, Glahn J, Helfer C, Ickenstein G, Keller J, Lapa S, Ledl C, Lindner-Pfleghar B, Nabavi D, Prosiegel M, Riecker A, Stanschus S, Warnecke T, Busse O. FEES für neurogene Dysphagien. Nervenarzt 2014; 1-9. DOI 10.1007/so0115-0144114-7.

28. Ebihara S, Sekiya H, et al. Dysphagia, dystussia, and aspiration pneumonia in elderly people. J Thorac Dis 2016; 8(3): 632-9.

29. Ekberg O, Hrsg. Radiology of the pharynx and the esophagus. Berlin: Springer 2004.

30. Erdman J, Oria M, Pillsbury L edit. Nutrition and Traumatic Brain Injury: Improving Acute and Subacute Health Outcomes in Military Personnel. Committee on Nutrition, Trauma, and the Brain Food and Nutrition Board, Institute of Medicine of the National Academies. The National Academies Press Washington, 2011. www.nap.edu/catalog.php?record_id=13121, download 10.06.2016.

31. Fietzek U, Kossmehl P, Barthels A, Ebersbach G, Zynda $\mathrm{B}$, Wissel J. Botulinum toxin B increases mouth opening in patients with spastic trismus. Eur J Neurol 2009; 16: 1299-1304.

32. Fraser C, Power M, Hamdy S, Rothwell J, Hobday D, Hollander I et al. Driving Plasticity in Human Adult Motor Cortex is Associated with Improved Motor Function after Brain Injury. Neuron 2002; 34: 831-40.

33. Freed M, Freed L, Chatburn R, Christian M. Electrical stimulation for swallowing disorders caused by stroke. Respiratory Care 2001; 46: 466-74.

34. Gosney M, Martin MV, Wright AE. The role of selective decontamination of the digestive tract in acute stroke. Age and Ageing 2006; 35: 42-7.

35. Hamdy S, Aziz Q, Rothwell JC, Power M, Singh KD, Nicholson DA, Tallis RC, Thompson DG. Recovery of Swallowing After Dysphagic Stroke Relates to Functional Reorganisation in the Intact Motor Cortex. Gastroenterology 1998; 115(5): 1104-12.

36. Hammond FM, Horn SD, et al. Readmission to an Acute Care Hospital During Inpatient Rehabilitation for Traumatic Brain Injury. Archives of Physical Medicine and Rehabilitation 2015; 96(8 Suppl 3): S293-303.

37. Hannig C. Radiologische Funktionsdiagnostik des Pharynx und des Ösophagus. Berlin: Springer 1995.

38. Hansen TS, Larsen K, Engberg A. The Association of Funktional Oral Intake and Pneumonia in Patients with Severe Traumatic Brain Injury. Arch Phys Med Rehabil 2008; 89: 2114-20.

39. Heidler MD, Bidu L, et al. Oralisierung langzeitbeatmeter Patienten mit Trachealkanüle. Med Klein Intensivmed Notfmed 2015; 110: 55-60.

40. Hind JA, Gensler G, Brandt DK, Miller Gardner PJ, Blumenthal L, Gramigna GD, Kosek S, Lundy D, McGarvey-Toler S, Rockafello S, Sullivan PA, Villa M, Gill GD, Lindblad AS, Logemann JA, \& Robins J. Comparison of Trained Clinician Ratings with Expert Ratings of Aspiration on Videofluoroscopic Images from a Randomised Clinical Trial. Dysphagia 2009; 24 (2): 211-7.

41. Horn SD, Kinikini M, et al. Enteral Nutrition for Patients With Traumatic Brain Injury in the Rehabilitation Setting: Associations With Patient Preinjury and Injury Characteristics and Outcomes. Arch Phys Med Rehabil 2015; 96(8 Suppl 3): S245-55.

42. Ihde SK, Konstantinovic VS. The Therapeutic Use of Botulinum Toxin in Cervical and Maxillofacial Conditions: An Evidence-Based Review. Oral Surg Oral Med Oral Pathol Oral Radiol Endod 2007; 104(2): e1-11. 
43. Jayasekeran V, Singh S, Tyrrell P, Michou E, Jefferson S, Mistry S, Gamble E, Rothwell J, Thompson D, Hamdy S. Adjunctive Functional Pharyngeal Electrical Stimulation Reverses Swallowing Disability After Brain Lesions. Gastroenterology 2010; 138: 1737-46.

44. Johnson ER, McKenzie SW, Rosenquist CJ, Lieberman JS, Sievers AE. Dysphagia Following Stroke: Quantitative Evaluation of Pharyngeal Transit Times. Arch Phys Med Rehabil 1992; 73(5): 419-23.

45. Kahrilas P, Gosh S, Pandolfino J. Esophageal Motility Disorders in Terms of ressure Topography: The Chicago Classification. J Clin Gastroenterol 2008; 42(5): 627-35.

46. Kahrilas PJ, Logeman JA, Krugler C, Flanagan E. Volitional Augmentation of Upper Esophageal Sphinkter Opening During Swallowing. Am J Physiol 1991; 23: G450-6.

47. Kendall K, McKenzie S, Leonard R. Dynamic swallow study: objective measures and normative data in adults. In: Leonard R, Kendall K, Hrsg. Dysphagia Assessment and treatment planning. Abingdon: Plural Publishing 2008.

48. Kiger M, Brown C, Watkins. Dysphagia management: An Analysis of Patient Outcomes Using VitalStim Therapy Compared to Traditional Swallow Therapy. Dysphagia 2006; 21: 243-53.

49. Kojima C, Fujishima I, Ohkuma R, Maeda H, Shibamoto I, Hojo K, Arai M. Jaw Opening and Swallow Triggering Method for Bilateral-Brain-Damaged Patients: K-Point Stimulation. Dysphagia 2002; 17: 273-7.

50. Kommission für Krankenhaushygiene und Infektionsprävention (KRINKO). Prävention der nosokomialen beatmungsassoziierten Pneumonie. Bundesgesundheitsbl 2013; 56: 1578-90.

51. Kondrup J, Rasmussen HH, et al. Nutritional risk screening (NRS 2002): a new method based on an analysis of controlled clinical trials. Clin Nutr 2003; 22(3): 321-36.

52. Lakraj AA, Moghimi N, Jabbari Bahman. Sialorrhea: Anatomy, Pathophysiology and Treatment with Emphasis on the Role of Botulinum Toxins. Toxins; 2013; 5; 1010-31.

53. Langmore S, Schatz K, Olson N (1988). Fiberoptic Endoscopic Examination of Swallowing Safety: A New Procedure. Dysphagia 1988; 2: 216-19.

54. Langmore SE, Terpenning MS, Schork A, Chen Y, Murray JT, Lopatin DL, Loesche WJ. Predictors of Aspiration Pneumonia: How Important Is Dysphagia? Dysphagia 1998; 13: 69-81.

55. Leder SB. Gag reflex and dysphagia. Head and Neck 1996; 18(2): 138-41.

56. Ledl C, Heller C, Hinterberger K, Houamed M, Klabuschnig M. Are Screening Procedures Useful in Postacute Dysphagic Patients? Dysphagia 2014; 29(1): 143-4.

57. Ledl C. Diagnostik neurogener Schluckstörungen. Neuroreha 2013; 4: 160-9.

58. Ledl C. Does Pharyngeal Electrical Stimulation Improve Swallowing Function in Patients with Bihemispheric Lesions? In: Hömberg V, Binder H (eds.). 6th World Congress for Neurorehabilitation 2010; P170.

59. Ledl C. Schluckstörungen. In: Müller F, Walther E, Herzog J (Hrsg). Praktische Neurorehabilitation. Stuttgart: Kohlhammer 2014; 197-207.

6o. Logeman J. Evaluation and Treatment of Swallowing Disorders. Austin Texas: Pro-Ed; 1983.

61. Löser Ch. Malnutrition in hospital - the clinical and economic implications. Dtsch Arztebl Int 2010; 107(51-52): 911-7.

62. Ludlow C, Humbert I, Saxon K, Poletto C, Sonies B, Crujido L. Effects of Surface Electrical Stimulation Both at Rest and During Swallowing in Chronic Pharyngeal Dysphagia. Dysphagia 2007; 22: 1-10.

63. Macht M, King CJ, et al. Post-extubation dysphagia is associated with longer hospitalization in survivors of critical illness with neurologic impairment. Crit Care 2013; 17(3): R119.
64. Macht M, Wimbish T, et al. Postextubation dysphagia ist persistent and associated with poor outcomes in survivors of critical illness. Crit Care 2011; 15(5): R231.

65. Marian T, Dziewas R. Schlaganfallbedingte Dysphagie Epidemiologie, Pathophysiologie, Diagnostik und Therapie. Aktuel Ernahrungsmed 2016; 41: 21-6.

66. Martino R, Foley N, et al. Dysphagia after stroke: incidence, diagnosis and pulmonary complications. Stroke 2005; 36: 2756-63.

67. Martino R, Silver F, Teasell R, et al. The Toronto Bedside Swallowing Screening Test (TOR-BSST): development and validation of a dysphagia screening tool for patients with stroke. Stroke 2009; 40: 555-61.

68. Massanet PL, Petit L, et al. Nutrition Rehabilitation in the Intensive Care Unit. Journal of Parenteral and Enteral Nutrition 2015; 39(4): 391-400.

69. McClave SA, Taylor BE, et al. Guidelines for the Provision and Assessment of Nutrition Support Therapy in the Adult Critically Ill Patient: Society of Critical Care Medicine (SCCM) and American Society for Parenteral and Enteral Nutrition (A.S.P.E.N.). Journal of Parenteral and Enteral Nutrition 2016; 40(2): 159-211.

70. Miranda-Rius J, Brunet-Llobet L, et al. Salivary Secretory Disorders, Inducing Drugs, and Clinical Management. Int. J Med. Sci. 2015; 12(10): 811-24.

71. O’Neill-Pirozzi TM, Lisiecki DJ, Jack Momose K, Connors JJ, Milliner MP. Simultaneuos Modified Barium Swallow and Blue Dye Test: A Determination of the Accuracy of Blue Dye Test Aspiration Findings. Dysphagia 2003; 18(1): 32-8.

72. Ohmae Y, Logeman JA, Kaiser P, Hanson DG, Kahrilas PJ. Effects of Two Breath Holding Maneuvers on Pharyngeal Swallow. Ann Otol Rhinol Laryngol 1996; 105(2): 123-31.

73. Pandolfino J, Gosh Z, Zhang Q, Jarosz A, Shan N, Khahrilas P. Quantifying EGJ Morphology and Relaxation with High-Resolution Manometry: A Study of 75 Asymptomatic Volunteers. Am J Physiol Gastrointest Liver Physiol 2006; 290(5): G1033-40.

74. Pearlman A, Vandaele D, Ottenbacher M. Quantitative Assessment of Hyoid Bone Displacement from Video Images During Swallowing. J Speech Hear Res. 1995; 38: 579-85.

75. PisegnaJ, Kaneoka A, Pearson W, Kumar S, Langmore S. Effects of Non-Invasive Brain Stimulation on Post-Stroke Dysphagia: A Systematic Review and Meta-Analysis of Randomized Controlled Trials. Clin Neurophysiol 2016; 127(1): 956-68.

76. Pohl M, Bertram M et al. Rehabilitationsverlauf von Patienten in der neurologisch-neurochirurgischen Frührehabilitation. Nervenarzt 2016; 87(6): 634-44.

77. Ponfick M, Linden R, Nowak D. Dysphagia - a common, transient symptom in critical illness polyneuropathy: a fiberoptic endoscopic evaluation of swallowing study. Crit Care Med 2015; 43(2): 365-72.

78. Rasley A, Logeman JA, Kahrilas PJ, Rademaker AW, Pauloski BR, Dodds WJ. Prevention of Barium Aspiration During Videofluoroscopic Swallowing Studies: Value of Change in Posture. Am J Roentgenol 1998; 160(5): 1005-9.

79. Restivo D, Casabona A, Centonze D, Marchese-Ragona R, Maimone D, Pavone A. Pharyngeal Electrical Stimulation for Dysphagia Associated with Multiple Sclerosis: A Pilot Study. Brain Stimul 2013; 6(3): 418-23.

80. Rofes L, Arreola V, et al. Diagnosis and Management of Oropharyngeal Dysphagia and Its Nutritional and Respiratory Complications in the Elderly. Gastroenterology Research and Practice Volume 2011, Article ID 818979, 13 pages. doi:10.1155/2011/818979.

81. Rosenbek JC, Robbins JA, Roecker EB, Coyle JL, Wood JL. A Penetration-Aspiration Scale. Dysphagia 1996; 11(2): 93-98.

82. Rutte R, Sturm S. Atemtherapie, 2. Auflage. Heidelberg: Springer 2010. 
83. Schröter-Morasch H. Klinische Untersuchung des Oropharynx und videoendoskopische Untersuchung der Schluckfunktion. In: Bartholome G, Schröter-Morasch H, Hrsg. Schluckstörungen: Diagnostik und Rehabilitation. München: Urban \& Fischer 2010.

84. Schulman RC, Mechanick JI. Metabolic and Nutrition Support in the Chronic Critical Illness Syndrome. Respir Care 2012; 57(6): 958-77.

85. Scottish Intercollegiate Guidelines Network (SIGN). Brain Injury Rehabilitation in Adults. Edinburgh, 2013; No. 130. www.sign.ac.uk; download 24.04.2016.

86. Steele C, Cichero J. Physiological Factors Related to Aspiration Risk: A Systematic Review. Dysphagia 2014; 29: 295-304.

87. Stierwalt J, Youmans S. Tongue Measures in Individuals with Normal and Impaired Swallowing. Am J Speech Lang Pathol 2007; 16: 148-56.

88. Suiter D, Leder S. Clinical utility of the 3-ounce water swallow test. Dysphagia 2008; 23(3): 244-50.

89. Suiter D, Sloggy J, Leder S. Validation of the Yale Swallow Protocol: A Prosepective Double-Blinded Videofluoroscopic Study. Dysphagia 2014; 29: 199-203.

90. Suntrup S, Marian T, Schröder B, Suttrup I, Muhle P, Oelenberg S, Hamacher C, Minnerup J, Warnecke T, Dziewas R. Electrical Pharyngeal Stimulation for Dysphagia Treatment in Tracheotomized Stroke Patients: A Randomized Controlled Trial. Intensive Care Med 2015; 41(9): 1629-37.

91. Tan EK, Jankovic J. Treating Severe Bruxism with Botulinum Toxin. J Am Dent Assoc 2000; 131(2), 211-6.

92. Tanaka N, Nohara K, Kotani Y, Matsumura M, Sakai T. Swallowing frequency in elderly people during daily life. J Oral Rehabil 2013; epub, download 29.07.2013.

93. Teasell R, Foley N, et al. Dysphagia and Aspiration Following Stroke. Evidence-Based Review of Stroke Rehabilitation. www.ebrsr.com; download 24.4.2016.

94. Teramoto S, Fukuchi Y. Detection of Aspiration and Swallowing Disorder in Older Stroke Patients: Simple Swallowing Provocation Test versus Water Swallowing Test. Arch Phys Med Rehabil 2000; 81(11): 1517-9.

95. Trapl M, Enderle P, Nowotny M, Teuschl Y, Matz K, Dachenhausen A, Brainin M. Dysphagia bedside screening for acute-stroke patients: the Gugging Swallowing Screen. Stroke 2007; 38(11): 2948-52.

96. Waito A, Bailey GL, Molfenter SM, Zoratto DC, Steele CM Voice-quality abnormalities as a sign of dysphagia: validation against acoustic and videofluoroscopic data. Dysphagia 2011; 26(2): 125-34.

97. Warnecke T, Teismann I, Meimann W, et al. Assessment of aspiration risk in acute ischaemic stroke - evaluation of the simple swallowing provocation test. J Neurol Neurosurg Psychiatry 2008; 79: 312-4.

98. Warnecke T, Teismann I, Oelenberg S, Hamacher C, Ringelstein EB, Schäbitz WR, Dziewas R. The safety of fiberoptic endoscopic evaluation of swallowing in acute stroke patients. Stroke 2009; 40(2):482-6.

99. Welch-West P, Aubut JA, Foley N, Teasell R. EvidenceBased Review of Moderate to Severe Acquired Brain Injury: Part 5: Dysphagia \& Nutritional Interventions for Patients with Acquired Brain Injuries. www.abiebr.com, download 10.06.2016.

100. Wirth R, Dziewas R, et al. Leitlinie der Deutschen Gesellschaft für Ernährungsmedizin (DGEM) in Zusammenarbeit mit der GESKES, der AKE, der DGN und der DGG. Klinische Ernährung in der Neurologie - Teil des laufenden S3-Leitlinienprojekts Klinische Ernährung. Aktuel Ernahrungsmed Stand 2013. www.awmf.org/leitlinien/detail/11/073-020. html, download 10.06.2016.

101. Wuttge-Hannig A, Hannig C. Radiologische Funktionsdiagnostik von Schluckstörungen bei neurologischen Krankheitsbildern und bei therapierten onkologischen Kopf-
Hals-Erkrankungen. In: Bartholome G, Schröter-Morasch H, Hrsg. Schluckstörungen: Diagnostik und Rehabilitation. München: Urban \& Fischer 2010.

102. Zammit-Maempel I, Chapple CL, Leslie P. Radiation Dose in Videofluoroscopic Swallow Studies. Dysphagia 2007; 22: $13-5$.

103. Ziegler W. Speech Motor Control Is Task-Specific: Evidence from Dysarthria and Apraxia of Speech. Aphasiology 2003; 17(1): $3-36$.

104. Zielske J, Bohne S, et al. Dysphagie-Management im Akutund Langzeitverlauf bei kritisch kranken intensivpflichtigen Patienten. Med Klein Intensivmed Notfmed 2014; 109: $516-25$.

\section{Interessenvermerk}

Die Autoren geben an, dass kein Interessenkonflikt vorliegt.

Korrespondenzadressen:

Christian Ledl

Fachzentrum Neurologie

Neurophonetik und Schlucktherapie

Schön-Klinik Bad Aibling

Kolbermoorer Straße 72

83043 Bad Aibling

CLedl@Schoen-Kliniken.de

Dr. Marion Mertl-Rötzer

Fachzentrum Neurologie

Intensivmedizin

Schön-Klinik Bad Aibling

Kolbermoorer Straße 72

83043 Bad Aibling

MMertl-Roetzer@Schoen-Kliniken.de

Dr. Matthias Schaupp

Fachzentrum Neurologie

Neurologische Rehabilitation und Frührehabilitation

Schön-Klinik Bad Aibling

Kolbermoorer Straße 72

83043 Bad Aibling

MSchaupp@Schoen-Kliniken.de 